\title{
The Evolution of Portfolio Rules and the Capital Asset Pricing Model
}

\author{
Emanuela Sciubba* \\ Faculty of Economics and Politics \\ University of Cambridge
}

\begin{abstract}
The aim of this paper is to test the performance of the standard version of CAPM in an evolutionary framework. We imagine a heterogeneous population of long-lived agents who invest their wealth according to different portfolio rules and we ask what is the fate of those who happen to behave as prescribed by CAPM. In a complete securities' market with aggregate uncertainty, we prove that traders who either "believe" in CAPM and use it as a rule of thumb, or are endowed with genuine mean-variance preferences, under some very weak conditions, vanish in the long run. We show that a sufficient condition to drive CAPM or mean variance traders' wealth shares to zero is that an investor endowed with a logarithmic utility function enters the market. We finally check the robustness of our results allowing for different kinds of heterogeneity among traders.
\end{abstract}

JEL Classification C61, D81, G11

Keywords Evolution; portfolio rules; CAPM; Kelly criterion.

Address for Correspondence Emanuela Sciubba, Lucy Cavendish College, CB3 0BU Cambridge, UK. E-mail: es204@econ.cam.ac.uk; http://www.cus.cam.ac.uk/ es204.

\section{Introduction}

\subsection{Motivation}

A major part of the research in financial economics is directed towards improving our understanding of how investors make their portfolio decisions and hence of how asset prices are determined. Many capital asset-pricing models have been put forth in the literature. In particular, mean-variance analysis and the Sharpe-Lintner-Mossin CAPM ${ }^{1}$ are widely viewed as one of the "major contributions of academic research in the postwar era" [Jagannathan and Wang (1996), p.4].

* I am deeply indebted to Luca Anderlini for his helpful guidance. I also benefited from discussion with Robert Evans and Peter Sorensen. Useful comments came from participants to the St.John's Theory Workshops, University of Cambridge, to the Third International Conference on "Computation in Economics and Finance", Stanford University and to the Royal Economic Society 1999 Conference in Nottingham, UK. All remaining errors are mine.

${ }^{1}$ See Sharpe (1964), Lintner (1965) and Mossin (1996). 
Over the past two decades a number of studies have examined the empirical performance of CAPM, invariably providing strong evidence of its inability to explain (and therefore to predict) the behaviour of financial markets ${ }^{2}$. Nevertheless, "[i]n spite of the lack of empirical support, the CAPM is still the preferred model for classroom use in MBA and other managerial finance courses. In a way it reminds us of cartoon characters like Wile E. Coyote who have the ability to come back to original shape after being blown to pieces or hammered out of shape" [Jagannathan and Wang (1996), p.4].

In this paper we are after Wile E. Coyote once again, but with a new device. In fact, econometricians have empirically rejected its predictions and financial theorists have criticised its restrictive assumptions, but no one to our knowledge has studied CAPM in an evolutionary framework. The focus of our paper is to fill this gap in the literature and, in particular, to test the performance of the standard version of CAPM in an evolutionary setting.

Conventional financial theory shows that, under well-known assumptions, CAPM stems from rational behaviour. However, a recent strand of literature on evolution and market behaviour stresses that rationality is neither sufficient nor a necessary condition for survival. Therefore an interesting question to ask is whether CAPM prescribes a behaviour which can be considered "fit" in an evolutionary sense.

We imagine a heterogeneous population of long-lived agents who invest according to different portfolio rules and we ask what is the asymptotic market share of those who happen to behave as prescribed by CAPM. Namely we aim at detecting the asymptotic properties of the wealth shares of traders that either "believe" in CAPM and use it as a rule of thumb for their portfolio decisions, or display genuine mean-variance behaviour. Our results suggest that there are several circumstances of economic interest in which their wealth share will converge almost surely to zero. A sufficient condition to drive CAPM traders to extinction is that an investor endowed with a logarithmic utility function enters the market.

We believe that this is an interesting result not only because it proves that CAPM is not robust in an evolutionary sense, but also because it triggers once again the debate on the normative appeal and descriptive appeal of logarithmic utility approach as opposed to mean-variance approach in finance. Since a seminal article by Kelly (1956), several financial economists and applied probabilists have been debating whether maximising a logarithmic utility function is "more rational" for a rational trader. The debate originates from the dissatisfaction with the mean-variance approach which fails to single out a unique optimal portfolio. In fact, the chosen mix between the risk free asset and the market portfolio depends on each investor's degree of risk aversion. Several authors ${ }^{3}$ have argued that a rational long run investor should maximise the expected growth rate of his wealth share and, therefore, should behave as if he were endowed with a logarithmic utility function ${ }^{4}$ (the so called Kelly criterion). This yields a unique solution to the optimal portfolio problem. This claim has

\footnotetext{
${ }^{2}$ The empirical literature on CAPM is so vast that we will not attempt a review here. For a recent account, see, for istance, Jagannathan and Wang (1996) and Fama and French (1996a, 1996b).

${ }^{3}$ See, for example, Latane (1959), Breiman (1961), Mossin (1968), Hakansson (1971), Finkelstein and Whitley (1981) and Algoet and Cover (1988).

${ }^{4}$ Theoretical biologists [see Cooper and Kaplan (1982)] envisage an adaptive process which is similar to the Kelly criterion as the fittest reaction of animals to a changing (and therefore risky) environment.
} 
been opposed by Merton and Samuelson (1974) and Goldman (1974).

In particular, Merton and Samuelson's critique stressed the obvious contradiction which lies in arguing that rational traders should maximise a utility function which is different from their own ${ }^{5}$. The evolutionary framework adopted in this paper suggests that maximising a logarithmic utility function might not make you happy, but will definitely keep you alive!

\subsection{Related Literature}

The literature on evolution and market behaviour is in its relative infancy. The idea that economic institutions could be modeled as biological organisms is not at all new. As E. Penrose points out: "Economics has always drawn heavily on the natural sciences for analogies designed to help in the understanding of economic phenomena. Biological analogies in particular have been widely used in discussions of the firm" [E. Penrose (1952), p. 804]. However, the literature addressing producer $^{6}$ and consumer ${ }^{7}$ theory with evolutionary techniques has notably increased in recent years. The research effort in this direction has, in fact, become so impressive and diverse that we will not attempt a review here. We will, instead, concentrate on the more self-contained strand of literature to which the present paper specifically contributes. In particular, we will focus on the literature that aims at studying market forces as evolutionary forces and long run market outcomes as the result of a process akin to natural selection.

Most of the literature on evolution and market behaviour stems from the debate on bounded rationality in economics and finds motivation in the simple idea that individuals "may be irrational and yet markets quite rational" [G. Becker (1962), p. 8]. Becker proved that several basic features of orthodox economic theory can be derived as aggregate-level consequences of agents' behaviour, which need not to be rational in a strict sense and can, in fact, even be random. This insight has been often backed up by experimental evidence.

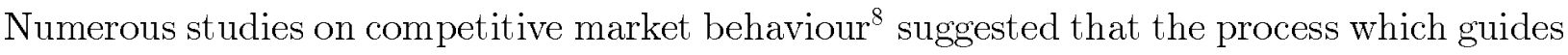
markets (near) to equilibrium does not rest upon agents' rationality.

A recent study by Luo (1995) proves that, in an evolutionary model of an industry where firms choose their output randomly, provided that there are no barriers to entry and that exit automatically occurs when a firm makes negative profits, the industry converges in probability to a perfectly competitive equilibrium.

Furthermore, a related strand of literature has been concentrating on noise trading. Noise traders in financial markets have been not only analysed from a welfare perspective, but also

\footnotetext{
${ }^{5}$ Another interesting contribution to this debate is due to Luenberger (1993). He develops a model where investors have preferences on infinite sequences of wealth rather than wealth at a fixed (but later taken to the limit) terminal time; he assumes that those preferences display a tail property, so that investors are interested only in the long run; and finally he shows that these asymptotic preferences can be represented by the expected logarithm of returns.

${ }^{6}$ Following Penrose (1952)'s seminal article, several authors have addressed the problem of firms' survival in an evolutionary setting. Both Witt (1986) and Schaffer (1989) test the common belief that profit maximising firms exibit superior survival performance.

${ }^{7}$ For instance, Rogers (1994) studies the evolution of time-preference by natural selection. A recent paper by Robson (1996) develops a biological model to determine the fittest attitude to risk.

${ }^{8}$ See Plott (1982) for a review of experimental results on competitive markets' behaviour. Interesting results on simulated financial markets are provided by Gode and Sunder (1993).
} 
from an evolutionary point of view. Shefrin and Statman (1994) develop a model in which sophisticated and informed Bayesian traders interact with traders that make systematic cognitive errors. They show that, provided that noise traders are patient enough and that they do not commit errors that are "too serious", they will not be driven to extinction by informed traders. De Long et al. $(1990,1991)$ prove that noise traders can eventually come to dominate the market, if they unwillingly happen to make "good" cognitive mistakes. Biais and Shadur (1994) consider a market where non-overlapping generations of buyers and sellers trade to share risk. They show that irrational traders, who misperceive the risk but enjoy a higher bargaining power, might outperform rational traders who correctly assess the distribution of risk.

This paper is very close in spirit to Blume and Easley $(1992,1993)$. They develop an evolutionary model of a financial market, identify conditions for survival and prove false the common belief that rational behaviour is always selected for and irrational behaviour is always selected against by market forces. In particular, they show that the fittest behaviour in a risky security market is prescribed by a logarithmic utility function ${ }^{9}$. Namely, traders who follow the Kelly criterion dominate and determine equilibrium prices asymptotically. On the other hand, whenever a logarithmic utility maximiser enters the market, all other types of traders are driven to extinction unless they asymptotically behave as if they were logarithmic utility maximisers. As a result, in the long run, traders who are endowed with a logarithmic utility function will survive, as well as successful imitators.

In this paper, we adopt Blume and Easley's framework and definitions of dominance, survival and extinction of traders. However, in order to show that logarithmic traders dominate and CAPM and mean-variance traders vanish, we cannot directly apply their results. This is because of two major reasons.

In the first place, Blume and Easley's results on logarithmic traders' dominance do not necessarily imply that CAPM traders would vanish. In fact, nothing in principle excludes that CAPM traders will asymptotically behave as logarithmic utility maximisers. This remark, which is certainly true for any general trading behaviour, is particularly biting for CAPM behaviour because of its imitative nature. In fact, a trader who believes in CAPM invests according to a risk-free and a market portfolio, where the most successful trading strategies are better represented. There is, therefore, some sort of imitative behaviour implicit in CAPM.

A second reason why our results do not stem from a direct application of Blume and Easley, is that both CAPM and mean-variance trading rules do not satisfy a crucial boundedness assumption which Blume and Easley impose. Their main theorem ${ }^{10}$, in fact, requires that the amount of wealth each trader invests in each asset has a uniform strictly positive lower bound. This technical assumption is not exactly harmless since it prevents us from applying their results to many interesting economic situations in which portfolio weights do not display uniform boundedness ${ }^{11}$ of this type. In our setting, for instance, it prevents us from comparing the relative fitness of CAPM and mean-variance behaviour as opposed to

\footnotetext{
${ }^{9}$ In a recent paper, Canning (1997) develops a model of selection of choice rules by means of imitation. He shows that the fittest choice rule is consistent with logarithmic utility maximisation.

${ }^{10}$ Theorem 4.1 in Blume and Easley (1992), p. 18.

${ }^{11}$ Without this boundedness assumption not even existence of equilibrium is guaranteed in their framework.
} 
logarithmic utility maximisation.

\subsection{Overview}

The structure of the paper is as follows. In section 2 we present the model and develop the evolutionary setting. Section 3 contains the main results for traders who believe in CAPM: first of all some trivial cases are discussed, then our main result is proved in a simple setting, and subsequently its robustness is checked removing some simplifying assumptions.

In particular, subsection 3.1 rules out two trivial special cases: in the absence of aggregate uncertainty and/or when CAPM traders' degree of risk aversion is such that they invest all their wealth in the market portfolio, CAPM traders behave exactly as logarithmic utility maximisers and, as immediate consequence, they survive. Subsection 3.2 deals with existence of equilibrium in this simple economy: we show that there is no market clearing equilibrium in a population of traders who believe in CAPM; on the contrary, existence and uniqueness are guaranteed whenever both logarithmic utility maximisers and CAPM traders are present in the market. Equilibrium prices are then characterised. Subsection 3.3 contains the main results: we prove that logarithmic traders dominate, determine equilibrium prices asymptotically, and drive to extinction the population of CAPM traders. Subsection 3.4 extends these results to $n$ types of traders, to traders with heterogeneous risk attitudes and finally to traders with different savings rates.

In section 4 we prove our main result for traders who display a genuine mean-variance behaviour. Section 5 concludes the paper.

For ease of exposition, proofs are in the appendix.

\section{The Model}

Consider a complete securities market. Time is discrete and indexed by $t=0,1,2, \ldots$. There are $S$ states of the world indexed by $s=1,2, \ldots, S$, one of which will occur at each date. States follow an i.i.d. process with distribution $p=\left(p_{1}, p_{2}, \ldots, p_{S}\right)$ where $p_{s}>0$ for all $s$. Let $\Omega=\times_{\mathbf{0}}^{\infty}\{1,2, \ldots, S\}$ with representative element $\omega=\left(\omega_{0}, \omega_{1}, \ldots, \omega_{t}, \ldots\right)$ where $\omega_{t}$ is the state at date $t$. Define $\Omega^{t}=\times_{0}^{t}\{1,2, \ldots, S\}$ with typical element $\omega^{t}$ for each $t=0,1,2, \ldots$. Let $\Im$ denote the product- $\sigma$-field on $\Omega$; finally let $\Im_{t}$ denote the sub- $\sigma$-field $\sigma\left(\omega^{t}\right)$ of $\Im$, so that $\left\{\Im_{t}, t \geq 0\right\}$ is a natural filtration.

Asset $s \in\{1,2, \ldots, S\}$ pays $w_{s}>0$ when state $s \in\{1,2, \ldots, S\}$ occurs and 0 otherwise. At each date there is only one unit of each asset available, so that $w_{s t}=w_{s}$ will be the total wealth in the economy at date $t$ if state $s$ occurs. This wealth will be distributed among the traders proportionately according to the share of asset $s$ each trader owns. We assume, unless otherwise stated, that there is aggregate uncertainty so that $w_{s} \neq w_{z}$ for $s \neq z$ : as shown in section 3.1.1 the evolution of the system becomes trivial in absence of aggregate risk. Moreover, without loss of generality, we assume: $w_{1}<w_{2}<\ldots<w_{S}$. Let $\rho_{s t}$ be the market price of (one unit of) asset $s$ at date $t$.

There is a finite number of traders in this economy, indexed by $i \in\{1,2, \ldots, I\}$. At date $t$, trader $i$ invests his savings from time $t-1, w_{t-1}^{i}$, in the $S$ available assets. He takes market 
prices as given and chooses a portfolio $\left\{q_{s t}^{i}\right\}_{s=1}^{S}$ where $q_{s t}^{i}$ denotes his demand of (shares ${ }^{12}$ of) asset $s$ at time $t$. It is often convenient to express his portfolio choice as a vector of portfolio weights $\left\{\alpha_{s t}^{i}\right\}_{s=1}^{S}$ where $\alpha_{s t}^{i}$ denotes the percentage of wealth available for investment at time $t$ for trader $i, w_{t-1}^{i}$, that he invests in asset $s$. Clearly, by definition, the following relation holds:

$$
\alpha_{s t}^{i} \equiv \frac{q_{s t}^{i} \rho_{s t}}{w_{t-1}^{i}}
$$

Call $r_{s t}^{i}$ trader $i$ 's investment income at date $t$ if state $s$ occurs and denote by $\delta_{s t}^{i}$ trader $i$ 's savings rate, defined as the percentage $r_{s t}^{i}$, saved for investment at date $t+1$ :

$$
\delta_{s t}^{i}=\frac{w_{t}^{i}}{r_{s t}^{i}}
$$

In order to ensure that traders always have positive wealth, we assume that every agent invests a strictly positive amount of savings in each of the available assets ${ }^{13}$, so that $q_{s t}^{i}$, $\alpha_{s t}^{i}>0, \forall i, \forall s, \forall t$. For the same reason, we assume that trader $i$ 's savings rate $\delta_{s t}^{i}$ is such that $\delta_{s t}^{i} \in(0,1], \forall i, \forall s, \forall t$.

Finally let $\alpha_{t}^{i} \equiv\left(\alpha_{1 t}^{i}, \alpha_{2 t}^{i}, \ldots, \alpha_{S t}^{i}\right), \delta_{t}^{i} \equiv\left(\delta_{1 t}^{i}, \delta_{2 t}^{i}, \ldots, \delta_{S t}^{i}\right)$ and $\rho_{t} \equiv\left(\rho_{1 t}, \rho_{2 t}, \ldots, \rho_{S t}\right)$; we refer to $\left\{\alpha_{t}^{i}\right\}_{t=1}^{\infty}$ as trader $i$ 's portfolio rule and to the pair $\left\{\alpha_{t}^{i}, \delta_{t}^{i}\right\}_{t=1}^{\infty}$ as trader $i$ 's investment rule.

Recall that we defined $w_{t-1}^{i}$ as the wealth that is available for investment in period $t$ for trader $i$, after his savings decision; therefore, by definition, it will be entirely invested in the available assets, so that $\sum_{s=1}^{S} \alpha_{s t}^{i}=1, \forall i, \forall t$. Similarly: $\sum_{s=1}^{S} q_{s t}^{i} \rho_{s t}=w_{t-1}^{i}, \forall i, \forall t$. At an aggregate level, this implies:

$$
\sum_{s=1}^{S} \rho_{s t}=\sum_{i=1}^{I} w_{t-1}^{i}=w_{t-1}
$$

Equation 3 provides us with a convenient normalisation for prices. We can, in fact, call $\pi_{s t}$ the normalised market price of asset $s$ at date $t$ and define it as follows:

$$
\pi_{s t} \equiv \frac{\rho_{s t}}{\sum_{s=1}^{S} \rho_{s t}}=\frac{\rho_{s t}}{w_{t-1}}
$$

Finally define: $\pi_{t} \equiv\left(\pi_{1 t}, \pi_{2 t}, \ldots, \pi_{S t}\right)$.

In equilibrium, prices must be such that markets clear, i.e. total demand equals total supply:

$$
\sum_{i=1}^{I} \frac{\alpha_{s t}^{i} w_{t-1}^{i}}{\rho_{s t}}=1
$$

Rewriting equation 5 we get:

$$
\rho_{s t}=\sum_{i=1}^{I} \alpha_{s t}^{i} w_{t-1}^{i}
$$

\footnotetext{
${ }^{12}$ Since at each date there is only one unit of each asset available, traders' portfolios contain shares rather than units of assets.

${ }^{13}$ In this model, we are mostly interested in detecting the long run dynamics of wealth shares; therefore we would like to rule out the possibility of any trader going bankrupt in finite time.
} 
or, equivalently:

$$
\pi_{s t}=\sum_{i=1}^{I} \alpha_{s t}^{i} \frac{w_{t-1}^{i}}{w_{t-1}}
$$

Equation 7, which is central to the functioning of our model, shows us how market prices are related to wealth shares.



Figure 1: Time line.

\subsection{The Dynamics of Wealth Shares}

In this market there is a natural population dynamic that emerges from the process of wealth accumulation. In particular, the evolution of wealth shares endogenously determines an adaptive dynamic.

Trader $i$ 's wealth share at date $t$ is defined as follows:

$$
\varepsilon_{t}^{i} \equiv \frac{w_{t}^{i}}{w_{t}}
$$

Recall that $w_{t} \equiv \sum_{i} w_{t}^{i}$ denotes total wealth available for investment at time $t+1$; for example, if state $s$ occurs at time $t$, then $w_{t}$ will be equal to $\delta_{s t} w_{s t}$, where $\delta_{s t}$ is the market savings rate and represents the share of total asset payout $w_{s t}$ which is saved for investment:

$$
\delta_{s t} \equiv \sum_{i} \delta_{s t}^{i} \frac{\alpha_{s t}^{i} w_{t-1}^{i}}{\rho_{s t}}
$$

Using 8 and 9 and recalling that, if state $s$ occurs at date $t$, trader $i$ 's wealth available for investment at the beginning of date $t+1$ will be:

$$
w_{t}^{i}=\delta_{s t}^{i} \frac{\alpha_{s t}^{i} w_{t-1}^{i}}{\varrho_{s t}} w_{s t}
$$


we obtain that:

$$
\varepsilon_{t}^{i}=\frac{\delta_{s t}^{i} \alpha_{s t}^{i}}{\delta_{s t} \varrho_{s t}} w_{t-1}^{i}
$$

and, using our price normalisation:

$$
\varepsilon_{t}^{i}=\frac{\delta_{s t}^{i} \alpha_{s t}^{i}}{\delta_{s t} \pi_{s t}} \varepsilon_{t-1}^{i}
$$

Equation 12 clearly represents a fitness-monotonic dynamic: trader $i$ 's wealth share will increase if and only if he scores a payoff which is higher than the average population payoff. In fact, if state $s$ occurs at date $t, \pi_{s t}$ gives us a measure of the average population payoff. The fittest behaviour, is therefore, that which maximises the expected growth rate of wealth share accumulation. We are now ready to define a formal notion of "dominance" in our model. We borrow the following definition from Blume and Easley (1992).

Definition 1 (a) Trader $i$ dominates on the set of sample paths $Q \in \Im$ if :

$$
\lim _{t \rightarrow \infty} \inf \varepsilon_{t}^{i}>0 \text { a.s. on } Q
$$

(b) Trader $i$ survives on the set of sample paths $Q \in \Im$ if :

$$
\lim _{t \rightarrow \infty} \sup \varepsilon_{t}^{i}>0 \text { a.s. on } Q
$$

(c) Trader $i$ vanishes on the set of sample paths $Q \in \Im$ if :

$$
\lim _{t \rightarrow \infty} \sup \varepsilon_{t}^{i}=0 \text { a.s. on } Q \text {. }
$$

Therefore, in order to establish if trader $i$ dominates on a sample path $\omega=\left(\omega_{0}, \omega_{1}, \ldots, \omega_{t}, \ldots\right)$ we need to consider the asymptotic behaviour of his wealth share: namely we have to check whether it is bounded away from zero or not. Note that the wealth share of a dominating trader does not necessarily converge to 1 , as there might be other surviving traders.

Moreover, market dominance is clearly related to the process of price determination: differential rates of wealth accumulation increase the relative significance of some individuals in determining market outcomes and namely equilibrium prices. In particular, Blume and Easley justify their use of the word "dominates" as follows: "When savings rates are identical, a trader who dominates actually determines the price asymptotically. His wealth share need not converge to one because there may be other traders who asymptotically have the same portfolio rule, but prices adjust so that his conditional expected gains converge to zero" [Blume and Easley (1992), p. 19]. This consideration also justifies the distinction between dominance and mere survival.

Under the assumption that investment rules are measurable and satisfy a boundedness property, Blume and Easley (1992) find sufficient conditions for dominance, survival and extinction.

Assumption 1 For all $t$ and all $i, \alpha_{t}^{i}$ is $\Im_{t-1}$-measurable and $\delta_{s t}^{i}$ is $\Im_{t}$-measurable. 
Assumption 2 There exists a real number $\varphi>0$ such that, for all $i, \delta_{s t}^{i}, \alpha_{s t}^{i} \geq \varphi$ for all $s$ and $t$, a.s.

The dynamics of wealth shares is more easily described by introducing some additional notation: let $X_{t}^{i}=\sum_{s} \iota_{s t} \log \delta_{s t}^{i} \alpha_{s t}^{i}$ and $Y_{t}=\sum_{s} \iota_{s t} \log \delta_{s t} \pi_{s t}$ where $\iota_{s t}$ is the indicator function that is equal to 1 if state $s$ occurs at date $t$ and equal to 0 otherwise. The expected values of $X_{t}^{i}$ and $Y_{t}$ conditional on the information available at time $t-1$, can be computed as follows:

$$
\begin{aligned}
E\left[X_{t}^{i} \mid \Im_{t-1}\right] & =\sum_{s=1}^{S} p_{s} \log \delta_{s t}^{i} \alpha_{s t}^{i} \\
E\left[Y_{t} \mid \Im_{t-1}\right] & =\sum_{s=1}^{S} p_{s} \log \delta_{s t} \pi_{s t}
\end{aligned}
$$

$E\left[X_{t}^{i} \mid \Im_{t-1}\right]$ is a statistic that summarises information about trader $i$ 's investment rule at date $t$ : it can be interpreted as an index that tells us how good player $i$ is in maximising the expected growth rate of his wealth share ${ }^{14}$.

Similarly, we can interpret $E\left[Y_{t} \mid \Im_{t-1}\right]$ as an index that tells us how good players are "on average" in maximising the expected growth rate of their wealth share. In fact:

$$
\delta_{s t} \pi_{s t}=\sum_{i} \delta_{s t}^{i} \alpha_{s t}^{i} \varepsilon_{t-1}^{i}
$$

i.e. $\delta_{s t} \pi_{s t}$ is actually a weighted average across traders of $\delta_{s t}^{i} \alpha_{s t}^{i}$, where weights are given by wealth shares at the beginning of period $t$.

We are now ready to state the following:

Theorem 1 Under assumptions 1 and 2 ,

(a) Trader $i$ dominates on:

$$
\left\{\omega \in \Omega: \lim _{t \rightarrow \infty} \inf \sum_{\tau=1}^{t} E\left\{X_{\tau}^{i}-Y_{\tau} \mid \Im_{\tau-1}\right\}>-\infty\right\}
$$

and, if all traders have the same savings rate, then: $\lim _{t \rightarrow \infty} \pi_{t}=\alpha_{t}^{i}$ a.s.;

(b) Trader $i$ survives on:

$$
\left\{\omega \in \Omega: \lim _{t \rightarrow \infty} \sup \sum_{\tau=1}^{t} E\left\{X_{\tau}^{i}-Y_{\tau} \mid \Im_{\tau-1}\right\}>-\infty\right\}
$$

(c) Trader $i$ vanishes on:

$$
\left\{\omega \in \Omega: \lim _{t \rightarrow \infty} \sup \frac{\sum_{\tau=1}^{t} E\left\{Y_{\tau} \mid \Im_{\tau-1}\right\}}{\sum_{\tau=1}^{t} E\left\{X_{\tau}^{i} \mid \Im_{\tau-1}\right\}}<1\right\} .
$$

\footnotetext{
${ }^{14}$ Note that $E\left[X_{t}^{i} \mid \Im_{t-1}\right]$ as a function of $\left\{\alpha_{s t}^{i}\right\}_{s=1}^{S}$ is globally concave and attains a global maximum at $\alpha_{s t}^{i}=p_{s}, \forall s \in\{1,2, \ldots, S\}$.
} 
The intuition behind conditions (a), (b) and (c) in theorem 1 is as follows: the dominating traders are those who are relatively better than the others in maximising the expected growth rate of their wealth shares; this "ability" is, therefore, the fitness criterion the market selects for. Note that condition (c) implies that conditions (b) and, a fortiori, (a) fail. In addition, condition (c) puts a restriction on the rate at which $\pi_{t}$ and $\alpha_{t}^{i}$ diverge.

Theorem 1 also states that, if all traders have the same savings rate ${ }^{15}$, the dominating trader determines market prices asymptotically. His wealth share, on the other hand, need not converge to 1 because there might be other surviving traders.

A formal proof of theorem 1 can be found in Blume and Easley (1992). Here, we give an intuitive account of the key steps in their argument. Let us consider, for example, the dominance condition under the simplifying assumption that all traders have identical savings rates. What the theorem tells us is that if $\liminf _{t \rightarrow \infty} \sum_{\tau=1}^{t} E\left\{X_{\tau}^{i}-Y_{\tau} \mid \Im_{\tau-1}\right\}>-\infty$, then $\liminf \operatorname{in}_{t \rightarrow \infty} \varepsilon_{t}^{i}>0$ almost surely. In other words, trader $i$ dominates.

To understand this result intuitively recall that, if state $s$ occurs at date $t$, then:

$$
\varepsilon_{t}^{i}=\frac{\alpha_{s t}^{i}}{\pi_{s t}} \varepsilon_{t-1}^{i}
$$

so that we can write:

$$
\varepsilon_{t}^{i}=\prod_{\tau=1}^{t} \prod_{s=1}^{S} \iota_{s \tau} \frac{\alpha_{s \tau}^{i}}{\pi_{s \tau}} \varepsilon_{0}^{i}
$$

and finally:

$$
\begin{aligned}
E\left[\log \varepsilon_{t}^{i}\right] & =\sum_{\tau=1}^{t} \sum_{s=1}^{S} p_{s} \log \frac{p_{s}}{\pi_{s \tau}}-\sum_{\tau=1}^{t} \sum_{s=1}^{S} p_{s} \log \frac{p_{s}}{\alpha_{s \tau}^{i}}+\log \varepsilon_{\mathbf{0}}^{i}= \\
& =\sum_{\tau=1}^{t} E\left\{X_{\tau}^{i}-Y_{\tau} \mid \Im_{\tau-1}\right\}+\log \varepsilon_{\mathbf{0}}^{i}
\end{aligned}
$$

As a result, on those sample paths where $\liminf _{t \rightarrow \infty} \sum_{\tau=1}^{t} E\left\{X_{\tau}^{i}-Y_{\tau} \mid \Im_{\tau-1}\right\}>-\infty$, $\log \varepsilon_{t}^{i}$ is bounded away from $-\infty$ and therefore $\varepsilon_{t}^{i}$ is bounded away from 0 , which implies dominance of $i$ by definition.

\subsection{Types of Traders}

In our analysis we will consider three different types of traders.

The first type of agents is given by investors who believe in CAPM (type CAPM) and use it as a rule of thumb ${ }^{16}$ : at the beginning of each period, they observe payoffs and market prices and work out the composition of the market and the risk-free portfolios. Finally, according to their degree of risk aversion they choose their preferred combination between

\footnotetext{
${ }^{15}$ Blume and Easley (1992) also prove that the same result applies if traders do not have equal savings rates but $\delta_{s t}^{i} \geq \delta_{s t}$ a.s.

${ }^{16}$ We can think of them as of traders who have been educated in business schools. They have been taught the model so well in their finance courses that they believe it really works.
} 
the two. Index CAPM traders by $j$; at date $t$, investor $j$ chooses $\gamma_{t}^{j} \in[0,1]$ and invests in asset $s$ a portion $\alpha_{s t}^{C A P M(j)}$ of his wealth such that:

$$
\alpha_{s t}^{C A P M(j)}=\gamma_{t}^{j} \alpha_{s t}^{F}+\left(1-\gamma_{t}^{j}\right) \alpha_{s t}^{M}
$$

where:

$$
\alpha_{s t}^{F} \equiv \frac{\rho_{s t} / w_{s t}}{\sum_{z} \rho_{z t} / w_{z t}}=\frac{\pi_{s t} / w_{s t}}{\sum_{z} \pi_{z t} / w_{z t}}
$$

and

$$
\alpha_{s t}^{M} \equiv \frac{\rho_{s t}}{\sum_{z} \rho_{z t}}=\pi_{s t}
$$

The second type of traders is given by investors who are endowed with a logarithmic utility function (type $L$ ) and who actually maximise the growth rate of their wealth share and invest according to a "simple" portfolio rule:

$$
\alpha_{s t}^{L}=p_{s}
$$

More generally, at each date $t$, a rational trader $i$ will choose $\left\{q_{s t}^{i}\right\}_{s=1}^{S}$ so as to maximise:

$$
\sum_{s=1}^{S} p_{s} u^{i}\left(q_{s t}^{i} w_{s}\right)
$$

subject to the constraint that investment expenditure at each date is less than or equal to the amount of wealth saved in the previous period. If $u^{i}(\cdot)$ is logarithmic, it follows that $q_{s t}^{L}=\left(p_{s} / \rho_{s t}\right) w_{t-1}^{L}$ and that ${ }^{17} \alpha_{s t}^{L}=p_{s}$.

Finally, we shall consider traders who display a genuine mean-variance behaviour (type $M V)$, namely traders endowed with a quadratic utility function:

$$
u(\widetilde{w})=\widetilde{w}-\frac{a}{2} \widetilde{w}^{2}
$$

where $^{18} a<1 / w_{S}$.

Substituting 24 into 23 and solving for $q_{s t}^{M V}$ using the first order conditions, we obtain:

$$
q_{s t}^{M V}=\frac{1}{a w_{s}}-\frac{k_{t}-a \varepsilon_{t-1}^{M V}}{a h_{t} p_{s} w_{s}^{2}} \pi_{s t}
$$

where:

$$
k_{t}=\sum_{s=1}^{S} \frac{\pi_{s t}}{w_{s}} \quad \text { and } \quad h_{t}=\sum_{s=1}^{S} \frac{\pi_{s t}^{2}}{p_{s} w_{s}^{2}}
$$

and $\varepsilon_{t-1}^{M V}$ is the wealth share of mean-variance traders at (the beginning of) date $t$. Therefore:

$$
\alpha_{s t}^{M V}=\frac{\pi_{s t}}{a \varepsilon_{t-1}^{M V} w_{s}}-\frac{k_{t}-a \varepsilon_{t-1}^{M V}}{a h_{t} p_{s} w_{s}^{2} \varepsilon_{t-1}^{M V}} \pi_{s t}^{2}
$$

Clearly, if $\pi_{s t} \rightarrow 0$ for some $s$, then both $\alpha_{s t}^{C A P M} \rightarrow 0$ and $\alpha_{s t}^{M V} \rightarrow 0$ so that theorem 1 in section 2.1 [i.e. Theorem 4.1 of Blume and Easley (1992), p.18] does not apply.

\footnotetext{
${ }^{17}$ Note that we are assuming that traders know the probability distribution $p$ over the state space $S$. In a more general framework, a trader who displays a logarithmic utility function bets his beliefs.

${ }^{18}$ This bound guarantees that traders do not attain satiation.
} 


\section{Dynamics with Traders who Believe in CAPM}

Suppose that there are only two types of traders in the economy: traders who believe in CAPM and traders who are endowed with a logarithmic utility function. As usual, denote by $q_{s t}^{i}>0$ the quantity (share) of each asset $s$ that trader $i$ demands at time $t>0$. Denote, as in the more general case, total wealth available for investment at time $t$, by $w_{t-1}$. Finally, denote by $\varepsilon_{t-1}$ the share of aggregate wealth $w_{t-1}$ which belongs to type $L$, and by $\left(1-\varepsilon_{t-1}\right)$ the fraction of $w_{t-1}$ which belongs to type $C A P M$ at the beginning of period $t$.

As a temporary simplifying assumption, we will assume that the degree of risk aversion is homogeneous in the population of traders who believe in CAPM, so that $\gamma_{t}^{j}=\gamma_{t} \in[0,1]$, $\forall j, \forall t$ and, consequently, $\alpha_{s t}^{C A P M(j)}=\alpha_{s t}^{C A P M}, \forall j, \forall s, \forall t$.

In this section we will analyse both the static equilibrium of this simple economy and the intertemporal dynamic of wealth shares and prices. Before proceeding any further, we rule out two special cases that yield trivial results.

\subsection{Trivial Cases}

\subsubsection{No Aggregate Risk}

If there is no aggregate uncertainty, i.e. if $w_{s t}=w_{s^{\prime} t}, \forall s, s^{\prime} \in S$, we can state the following:

Remark 1 With no aggregate risk, in a population of traders who believe in CAPM and traders with logarithmic utility function, the behaviour of traders who believe in CAPM and traders with a logarithmic utility function coincides. Formally, if $w_{s t}=w_{s^{\prime} t}, \forall t \geq 0, \forall s, s^{\prime} \in$ $\{1, \ldots, S\}$, then $\alpha_{s t}^{C A P M}=p_{s}, \forall t>0, \forall s \in\{1, \ldots, S\}$.

The proof of remark 1 is straightforward. The intuition is that in the absence of aggregate risk, market and risk-free portfolio coincide, therefore traders who believe in CAPM actually invest only according to the market portfolio, so that their behaviour is purely imitative. As a result, when a logarithmic utility maximiser enters the economy, everyone invests according to his portfolio rule.

\subsubsection{Constant Absolute Risk Aversion}

The degree of risk aversion affects the choice of $\gamma_{t} \in[0,1]$. We will assume throughout that all investors are risk averse. If traders who believe in CAPM are very risk averse, they will invest most of their wealth in the riskless portfolio; as a result, a higher degree of risk aversion implies a larger value of $\gamma_{t}$.

We will examine the implications of how risk aversion varies with wealth in more detail in the following sections. Here we concentrate on a trivial case. In particular, assume that the degree of risk aversion does not change with wealth so that traders display constant absolute risk aversion. We can then state the following:

Remark 2 Under the CARA assumption, in a population of traders who believe in CAPM and traders with logarithmic utility function, if $\gamma_{t}=\gamma=0, \forall t$, the behaviour of traders who believe in CAPM and traders with a logarithmic utility function coincides, i.e. $\alpha_{s t}^{C A P M}=p_{s}$, $\forall s, \forall t$. 
Therefore traders who believe in CAPM will behave as logarithmic utility maximisers. On the other hand, it is straightforward to check that for any other value of $\gamma \neq 0$, it follows that $\alpha_{s t}^{C A P M} \neq p_{s}$ and therefore traders who believe in CAPM will behave differently from logarithmic utility maximisers.

\subsection{Existence of Equilibrium}

Consider a population where there are two types of traders: traders who believe in CAPM and traders who are endowed with a logarithmic utility function. Their wealth shares at (the beginning of) date $t$ are respectively $\left(1-\varepsilon_{t-1}\right)$ and $\varepsilon_{t-1}$. We will show that, at each date $t \geq 0$, a market clearing equilibrium exists, provided that $\varepsilon_{t-1}>0$ which, in turn, is guaranteed for all $t \geq 1$ provided that $\varepsilon_{0}>0$.

Traders' demands ${ }^{19}$ are as follows:

$$
\begin{aligned}
q_{s t}^{L} & =\frac{p_{s} \varepsilon_{t-1} w_{t-1}}{\rho_{s t}} \\
q_{s t}^{C A P M} & =\left(1-\varepsilon_{t-1}\right) \gamma_{t} \frac{w_{t-1}}{w_{s} \sum_{z} \rho_{z t} / w_{z}}+\left(1-\varepsilon_{t-1}\right)\left(1-\gamma_{t}\right)
\end{aligned}
$$

Recall that at each date there is only one unit available of each asset, so that market clearing requires:

$$
q_{s t}^{L}+q_{s t}^{C A P M}=1, \quad \forall s \in\{1,2, \ldots, S\}
$$

which implies:

$$
\rho_{s t}=\frac{p_{s} \varepsilon_{t-1} w_{t-1} w_{s} \sum_{z} \rho_{z t} / w_{z}}{\left[1-\left(1-\varepsilon_{t-1}\right)\left(1-\gamma_{t}\right)\right] w_{s} \sum_{z} \rho_{z t} / w_{z}-\left(1-\varepsilon_{t-1}\right) \gamma_{t} w_{t-1}}
$$

We can show that there exists a unique non-negative price vector that satisfies 31 for all $s \in\{1, \ldots, S\}$ and therefore a unique market clearing equilibrium.

Definition 3 Market clearing equilibrium at date $t$ for this economy is an array of portfolios and assets' prices $\left\{\left(q_{s t}^{L}, q_{s t}^{C A P M}\right), \rho_{s t}\right\}_{s=1}^{S}$ such that, $\forall s \in\{1,2, \ldots, S\}$ :

- $q_{s t}^{L}$ satisfies 28 ;

- $q_{s t}^{C A P M}$ satisfies 29 ;

- $q_{s t}^{L}+q_{s t}^{C A P M}=1$;

- prices are non-negative: $\rho_{s t} \geq 0$.

Proposition 2 Provided that $\varepsilon_{0}>0$, at each date $t \geq 0$ there exists a unique market clearing equilibrium.

\footnotetext{
${ }^{19}$ Note that trader $i$ 's demand of asset $s$ at date $t$ is equal to:

$$
q_{s t}^{i}=\frac{\alpha_{s t}^{i} w_{s t}^{i}}{\rho_{s t}}
$$
}

where $\alpha_{s t}^{i}$ is trader $i$ 's portfolio weight in asset $s$. 
Proof. See appendix.

An immediate corollary of equation 31 is that there is no market clearing equilibrium if all traders behave according to CAPM rule. A clear intuition for this result is that in such an economy every trader would like to invest his whole wealth in the risk-free portfolio. However, in a risky world, not everybody can be fully insured: as long as there is aggregate uncertainty, for an equilibrium to exist some traders must bear the risk.

On the other hand, a unique equilibrium always exists in an economy populated only by traders who are endowed with a logarithmic utility function. In such a world, at each date, (normalised) equilibrium prices are equal to probabilities: $\pi_{s t}=p_{s} \forall s, t$.

We can characterise the limiting behaviour of prices as $\varepsilon_{t} \rightarrow 0$. When the wealth share of logarithmic utility maximisers approaches zero, equilibrium prices move towards a vertex of the price simplex. In particular, in the limit, only the market of asset 1 (in our notation, the asset with the lowest payout) clears with a strictly positive price.

Proposition 3 When $\varepsilon_{t} \rightarrow 0, \pi_{1 t} \rightarrow 1$ while $\pi_{s t} \rightarrow 0, \forall s \in\{2, \ldots, S\}$. In compact notation:

$$
\lim _{\varepsilon_{t} \rightarrow \mathbf{0}} \pi_{t}=\left[\begin{array}{c}
1 \\
0 \\
\vdots \\
0
\end{array}\right]
$$

Proof. See appendix.

In fact, in the limit, non-negativity of prices requires $\sum_{s=1}^{S} \pi_{s t} / w_{s} \geq 1 / w_{1}$, while market clearing requires $\sum_{s=1}^{S} \pi_{s t} / w_{s} \leq 1 / w_{1}$. The unique limiting value for $\sum_{s=1}^{S} \pi_{s t} / w_{s}$ that satisfies both is:

$$
\lim _{\varepsilon_{t} \rightarrow \mathbf{0}} \sum_{s=1}^{S} \frac{\pi_{s t}}{w_{s}}=\frac{1}{w_{1}}
$$

which, in turn, implies ${ }^{20} \lim _{\varepsilon_{t} \rightarrow \mathbf{0}} \pi_{t}=(1,0, \ldots, 0)$.

We can, finally, characterise traders' demands as $\varepsilon_{t} \rightarrow 0$.

\section{Corollary 4}

$$
\begin{aligned}
\lim _{\varepsilon_{t} \rightarrow \mathbf{0}} q_{s t}^{L} & =\gamma_{t}-\gamma_{t} \frac{w_{1}}{w_{s}} \\
\lim _{\varepsilon_{t} \rightarrow \mathbf{0}} q_{s t}^{C A P M} & =1-\gamma_{t}+\gamma_{t} \frac{w_{1}}{w_{s}}
\end{aligned}
$$

Proof. See appendix.

Notice that $\lim _{\varepsilon_{t} \rightarrow \mathbf{0}}\left(q_{s t}^{L}+q_{s t}^{C A P M}\right)=1$, so that there is market clearing $\forall s \in\{1, \ldots, S\}$. Both types of traders invest in assets $s \in\{2, \ldots, S\}$; only CAPM traders invest in asset 1 .

\footnotetext{
${ }^{20}$ The fact that all prices, except the price of the asset with the lowest payout, go to zero might seem counter-intuitive. However, consider that, as $\varepsilon_{t} \rightarrow 0$, we are left with an economy which is (almost) entirely populated by traders who invest their (almost) whole wealth in the risk-free portfolio. In order to insure themselves against aggregate risk, traders invest comparatively more in assets with lower payouts and higher prices. As a result, the aggreate demand for the asset with the lowest payout increases as $\varepsilon_{t} \rightarrow 0$, while demand for the other assets decreases.
} 
An immediate consequence of proposition 3 is that portfolio weights of traders who believe in CAPM are not bounded away from zero on those sample paths where $\varepsilon_{t} \rightarrow 0$. As a result, theorem 1 by Blume and Easley does not apply. In particular, we cannot use it to show that logarithmic traders dominate, since we would need to assume their dominance (and namely that $\varepsilon_{t} \nrightarrow 0$ ) in order to apply the theorem.

\subsection{The Main Result}

In this section we prove our results under a simplifying assumption which we will remove in the following sections. In particular, in order to concentrate on portfolio rather than on investment rules, we will assume that, almost surely, at every date and in every state of nature, logarithmic utility maximisers and traders who believe in CAPM display the same savings rate.

Assumption 3 For all $i \in\{L, C A P M\}$, all $s \in\{1, \ldots, S\}$ and all $t>0, \delta_{s t}^{i}=\delta_{s t}$ a.s.

We present our main result as two separate propositions. The first one (proposition 5) claims that in a population of traders who believe in CAPM and traders who are endowed with a logarithmic utility function, the latter dominate almost surely. This result yields, as main implication, that equilibrium prices converge a.s. to probabilities. The second proposition (proposition 7) claims that in a population of traders who believe in CAPM and traders who are endowed with a logarithmic utility function, in the presence of aggregate uncertainty (and unless we are in the CARA special case of remark 2), the former vanish almost surely. In fact, the wealth share of traders who believe in CAPM converges a.s. to zero: market forces drive them to extinction.

The first of these two results accords with Blume and Easley (1992): logarithmic utility maximisers dominate and determine equilibrium prices asymptotically. This clearly backs up the intuition that the fitness criterion market selects for is the "ability" to maximise the expected growth rate of wealth shares. The fittest portfolio rule is, indeed, the one adopted by logarithmic utility maximisers.

Proposition 5 Under assumptions 1 and 3, in a population of traders who believe in CAPM and traders who are endowed with a logarithmic utility function, the latter dominate almost surely. Formally: $\liminf \operatorname{in}_{t \rightarrow \infty} \varepsilon_{t}>0$ a.s.

Proof. See appendix.

The proof of proposition 5 consists of two steps. We first show that the evolution of logarithmic utility maximisers' wealth share:

$$
\varepsilon_{t}=\frac{p_{s}}{\pi_{s t}} \varepsilon_{t-1}
$$

follows a submartingale, so that for each date $t>0, E\left[\varepsilon_{t} \mid \mathfrak{F}_{t-1}\right] \geq \varepsilon_{t-1}$. This result (lemma 19 in the appendix) allows us to claim that $\varepsilon_{t}$ will converge almost surely to some value $\varepsilon^{*}$. Finally, we prove that, in presence of aggregate uncertainty, $\varepsilon^{*}(\omega) \neq 0$ a.s., so that $\lim _{t \rightarrow \infty} \varepsilon_{t}>0$ almost surely and, by definition, logarithmic utility maximisers dominate.

The next proposition claims that an important implication of logarithmic utility maximisers' dominance is that they determine equilibrium prices asymptotically. 
Proposition 6 Under assumptions 1 and 3, in a population of traders who believe in CAPM and traders who are endowed with a logarithmic utility function, the latter determine asset prices asymptotically, so that, $\forall s \in\{1, \ldots, S\}$ :

$$
\pi_{s t} \rightarrow p_{s} \quad \text { a.s. }
$$

Proof. See appendix.

This result is an immediate consequence of the fact that the wealth share of traders who are endowed with a logarithmic utility function is bounded away from zero almost surely. Note that the converse is not true: namely, the fact that prices converge to probabilities does not guarantee that the wealth share of logarithmic utility maximisers is bounded away from zero ${ }^{21}$. It is in fact possible that:

$$
\frac{\varepsilon_{t}}{\varepsilon_{t-1}}=\frac{p_{s}}{\pi_{s t}} \rightarrow 1
$$

and yet $\varepsilon_{t} \rightarrow 0$.

Fxtinction of traders who believe in CAPM is a result that one could not directly anticipate through Blume and Easley's theorem 1. For example, a very sensible hypothesis could have been that traders who use CAPM as a rule of thumb survive because they asymptotically behave as logarithmic utility maximisers. As a matter of fact, we have already examined two trivial cases $^{22}$ in which this actually happens not only asymptotically, but at every date. What we need to prove here is that the imitative behaviour implicit in CAPM is not powerful enough to prevent traders from vanishing.

In order to prove the next result, we need to make a further assumption ${ }^{23}$ on traders' behaviour towards risk.

Assumption 4 The portion of wealth that traders who believe in CAPM decide to invest in the risk free portfolio, $\gamma_{t}$, is a monotonic (increasing or decreasing) function of their level of wealth, $w_{t-1}^{C A P M}$.

Proposition 7 Under assumptions 1, 3 and 4 and in presence of aggregate uncertainty, in a population of traders who believe in CAPM and traders who are endowed with a logarithmic utility function, the former vanish almost surely. Namely: $\lim _{t \rightarrow \infty} \varepsilon_{t}=1$, almost surely.

Proof. See appendix.

Intuitively, the proof of proposition 7 runs as follows. Dominance of logarithmic utility maximisers and price convergence to probabilities requires that in the long run all surviving

\footnotetext{
${ }^{21}$ One could also directly prove price convergence to probabilities through lemma A.1 in Blume and Easley (1992), p. 34. However, as we noted above, this would neither imply that logarithmic utility maximisers dominate, nor directly result in CAPM traders' extinction. The proof of proposition 6 which we provide in the appendix is shorter and more straightforward than the one implicit in Blume and Easley (1992). Moreover, we believe that the economic intuition behind proposition 6 is better captured by our proof, which shows how market dominance implies equilibrium price determination in the long run.

${ }^{22}$ See section 3.1 .

${ }^{23}$ We believe that assumption 4 could be further relaxed: it constitutes a sufficient but not necessary condition for proposition 7 to hold.
} 
traders invest according to the Kelly criterion. We prove that the CAPM rule does not succeed in fully imitating the behaviour of $L$ traders, so that CAPM traders do not survive.

To characterise the long run behaviour prescribed by CAPM, we look at the asymptotic weights in the market and in the risk-free portfolios. We find that the market portfolio weights converge a.s. to probabilities; the risk-free portfolio weights, on the contrary, converge a.s. to values which are different from probabilities if there is aggregate uncertainty.

The second step of the proof shows that, under assumption 4, there is no sample path for $\gamma_{t}$ such that CAPM traders asymptotically invest only according to the market portfolio. As a result, CAPM traders are driven to extinction.

\subsection{Extensions}

We proved proposition 5 and proposition 7 under some simplifying assumptions. In what follows, our aim is to check the robustness of our main results, namely the dominance of logarithmic utility maximisers and the extinction of traders who believe in CAPM, in three more general settings, allowing for various kinds of heterogeneity among traders.

In the first place, we test whether the same result obtains when we consider a diversified environment where in addition to logarithmic utility maximisers and traders who believe in CAPM there are other types of traders as well.

Secondly, we remove our initial simplifying assumption on identical preferences within the population of traders who believe in CAPM and ask whether our results still obtain when we consider heterogeneous risk attitudes.

Finally, we check to what extent our previous analysis holds when heterogeneous savings rates across traders are allowed.

\subsubsection{A Multipopulation Model}

Consider a richer environment where there are $N+2$, with $0<N<\infty$, types of traders: traders who believe in CAPM, traders with logarithmic utility function and, finally, $N$ other types of traders who behave according to other portfolio rules that a.s. do not converge to any of the previous two.

As in the two population framework, the trader with logarithmic utility function will dominate and prices will eventually converge a.s. to probabilities. Moreover, if we exclude special cases, dominance of $L$ traders drives to extinction traders who believe in CAPM, no matter which other types of traders might be around in the market.

Consider a population of traders who believe in CAPM and suppose that a trader with logarithmic utility function enters the market along with $N$ other types of traders with portfolio rules $\left\{\alpha_{s t}^{n}\right\}_{s=1}^{S}$ and $n=1,2, \ldots, N$. For simplicity we also assume that:

Assumption $5 \exists \varphi>0 \mid \alpha_{s t}^{n} \geq \varphi, \forall s, t, n$ a.s.

Assumption $6 \forall n \in\{1, \ldots, N\}, \forall s \in\{1, \ldots S\}$, $\alpha_{s t}^{n}$ converges to $p_{s}$ or to $\alpha_{s t}^{C A P M}$ with probability 0: $\operatorname{Pr}\left\{\omega \in \Omega \mid \alpha_{s t}^{n} \rightarrow p_{s}\right\}=0$ and $\operatorname{Pr}\left\{\omega \in \Omega \mid \alpha_{s t}^{n} \rightarrow \alpha_{s t}^{C A P M}\right\}=0$.

The first assumption is technical: it is the same assumption that Blume and Easley (1992) impose on each trader's investment rules and allows us to apply corollary 4.1 in Blume and Easley (1992), p. 20. The second assumption requires that the other $N$ traders' 
portfolio rules almost surely do not converge either to logarithmic utility maximisers' or to CAPM traders' portfolio rules. Assumption 6 is without loss of generality: suppose that with positive probability, for some $n$, either $\alpha_{t}^{n} \rightarrow p$ or $\alpha_{t}^{n} \rightarrow \alpha_{t}^{C A P M}$, then on those sample paths where this happens, all the results in this section would still apply by propositions 5 , 6 and 7. Therefore one could ideally split the sample space in two sets: the set of sample paths where assumption 6 holds and the set of sample paths where it doesn't, and still the propositions in this section would hold on the whole sample space.

Denote by $\varepsilon_{t}^{L}$ and $\varepsilon_{t}^{C A P M}$ the wealth shares at (the end of) date $t$ of traders with logarithmic utility function and of traders who believe in CAPM respectively. Denote by $\varepsilon_{t}^{n}$ the wealth share of traders of type $n$, for $n \in\{1, \ldots, N\}$. Clearly:

$$
\sum_{n=1}^{N} \varepsilon_{t}^{n}=1-\varepsilon_{t}^{L}-\varepsilon_{t}^{C A P M}, \forall t \geq 0 .
$$

It is possible to show ${ }^{24}$ that, provided that $\varepsilon_{0}^{L}>0$, then a market clearing equilibrium exists at each date. In particular, as $\varepsilon_{t}^{L} \rightarrow 0$, equilibrium prices $\pi_{s t} \rightarrow 0$ for some $s$ and therefore portfolio rules $\alpha_{s t}^{C A P M} \rightarrow 0$ for some $s$, so that, despite assumption 5 , theorem 1 by Blume and Easley is not applicable ${ }^{25}$.

However, we can extend to this multipopulation framework the result of proposition 5:

Proposition 8 Under assumptions 1, 3 and 5, given a population of traders who believe in CAPM, suppose that a trader with logarithmic utility function and $N$ other traders, with portfolio rules $\alpha_{s t}^{n}$ and $n=1, \ldots, N$, enter the market. Traders endowed with a logarithmic utility function will dominate almost surely and determine asset prices asymptotically.

Proof. See appendix.

The proof of proposition 8 is in three steps. We first show that logarithmic utility maximisers outperform each of the $N$ new types of traders. In fact, for all $n$, the ratio between of the wealth share of trader $n$ and the wealth share of $L O G$ traders converges almost surely to zero. We then prove that $L O G$ traders dominate by similar arguments to those used for proposition 5. Finally dominance of logarithmic utility maximisers implies that prices converge a.s. to probabilities.

Our next proposition shows that when logarithmic traders dominate, extinction of CAPM traders is "generic" in a sense to be discussed below. In particular, CAPM traders will survive only if the other portfolio rules represented in the market are such that all traders, in the aggregate, asymptotically behave as logarithmic utility maximisers.

Let $\varepsilon^{L *}, \varepsilon^{C A P M *}$ and $\varepsilon^{n *}$ denote the limiting values ${ }^{26}$ of $\varepsilon_{t}^{L}, \varepsilon_{t}^{C A P M}$ and $\varepsilon_{t}^{n}$ respectively, as $t \rightarrow \infty$. We can then state the following:

\footnotetext{
${ }^{24}$ The proof is similar to the proof of proposition 2 and is therefore omitted.

${ }^{25}$ The result $\lim _{\varepsilon_{t}^{L} \rightarrow 0} \pi_{s t}=0$ stems from the fact that logarithmic utility maximisers dominate each of the $N$ populations of traders [corollary 4.1 in Blume and Easley (1992), p. 20]. Therefore, when the wealth share of logarithmic utility maximisers goes to zero, a fortiori the wealth shares of the other $N$ populations go to zero (see also the proof of proposition 8), so that the economy is only left with CAPM traders.

${ }^{26}$ We proved that $\varepsilon_{t}^{L} \rightarrow \varepsilon^{L *}$ a.s., so that such a limiting value for $L$ traders' wealth share exists. We will show that $\varepsilon_{t}^{C A P M}$ and $\varepsilon_{t}^{n}, \forall n$, almost surely converge to some values as well.
} 
Proposition 9 Under assumptions 1, 3, 4, 5 and 6, given a population of traders who believe in CAPM, suppose that a trader with logarithmic utility function and $N$ other traders, with portfolio rules $\alpha_{s t}^{n}$, where $n=1, \ldots, N$, enter the market. Unless the evolution of the system is such that, $\forall s \in\{1, \ldots, S\}$ :

$$
\sum_{n=1}^{N} \varepsilon^{n *} \alpha_{s t}^{n} \rightarrow p_{s}\left(1-\varepsilon^{L *}\right)-\varepsilon^{C A P M *} \alpha_{s t}^{C A P M} \text { a.s. }
$$

traders who believe in CAPM vanish. Formally: $\lim _{t \rightarrow \infty} \varepsilon_{t}^{C A P M}=0$ almost surely.

Proof. See appendix.

Note that the condition 36 in proposition 9 can also be expressed as follows:

$$
\frac{\sum_{n=1}^{N} \varepsilon^{n *} \alpha_{s t}^{n}+\varepsilon^{C A P M *} \alpha_{s t}^{C A P M}}{1-\varepsilon^{L *}} \rightarrow p_{s} \text { a.s. }
$$

When this relation holds, even if none of the traders asymptotically behaves as a logarithmic utility maximiser, the market as a whole does. In fact, what equation 36 requires is that the $N$ new rules represented in the market should complement $C A P M$ behaviour so that we could think of the $N$ traders together with $C A P M$ traders as of a single trader whose portfolio rules are asymptotically equal to probabilities. As a result, all traders survive.

This condition clearly imposes severe restrictions on the class of portfolio rules that should be represented in the market. For this reason, we claim that extinction of traders who behave as prescribed by CAPM is "generic". In fact, suppose we start with $N$ types of traders such that condition 36 holds. In case one of them, say trader $\hat{n}$, is replaced by a new type of trader with portfolio rule $\alpha_{s t}^{\hat{n}}=\alpha_{s t}^{n}+d_{s t}$ such that $\sum_{s} d_{s t}=0$, then condition 36 is not necessarily satisfied. Therefore survival of $C A P M$ traders is not robust to "small" changes to the set of the new $N$ types of traders introduced in the market.

\subsubsection{Heterogeneous Risk Attitudes}

Until now we have assumed that traders who believe in CAPM share the same degree of risk aversion. Namely, we have been considering traders with identical preferences that would choose the same combination of safe and risky portfolios. An interesting question to ask is whether our results are robust when we allow for heterogeneity in the degree of risk aversion among the population of traders who believe in CAPM.

We show that our results hold in this new setting. In fact, we can deal with heterogeneity thinking of a population of traders endowed with different degrees of risk aversion as of a single "average" trader whose portfolio rules are given by an appropriate weighted average of each trader's portfolio rules.

Consider a population of traders who believe in CAPM, indexed by $j \in\{1, \ldots, J\}$, and who have heterogeneous risk attitudes; trader $j$ 's portfolio rules at time $t$ will be:

$$
\alpha_{s t}^{C A P M(j)}=\gamma_{t}^{j} \alpha_{s t}^{F}+\left(1-\gamma_{t}^{j}\right) \alpha_{s t}^{M}
$$


where assumption 4 holds $^{27}$ for each $j \in\{1, \ldots, J\}$.

As usual we assume that a logarithmic utility maximiser enters the market with portfolio rule $\alpha_{s t}^{L}=p_{s}$. Denote by $\varepsilon_{t}$ and $\varepsilon_{t}^{j}$ the wealth shares at (the end of) date $t$ of $L O G$ traders and of CAPM trader $j$, respectively. We can claim the following:

Proposition 10 Under assumptions 1, 3 and 4, logarithmic utility maximisers dominate and drive to extinction a population of heterogeneous traders who believe in CAPM. Formally, $\limsup _{t \rightarrow \infty} \varepsilon_{t}^{j}=0$, for all $j \in\{1, \ldots, J\}$.

Proof. See appendix.

The proof of proposition 10 is in three steps. We first show that logarithmic utility maximisers dominate in a world of aggregate uncertainty. Again, an immediate corollary of this result is that prices converge a.s. to probabilities. The final part of the proof shows that, assuming that $\gamma_{t}^{j}$ is a monotonic (increasing or decreasing) function of wealth, $\forall j \in\{1, \ldots, J\}$ is a sufficient condition for all CAPM traders to vanish. In fact, dominance of logarithmic utility maximisers and price convergence to probabilities imply:

$$
\sum_{j=1}^{I-1} \varepsilon_{t-1}^{j} \gamma_{t}^{j} \rightarrow 0 \text { a.s. }
$$

and, since for each $j$ the set of sample paths where $\gamma_{t}^{j} \rightarrow 0$ has measure zero, condition 38 requires $\varepsilon_{t}^{j} \rightarrow 0$ a.s. for all $j \in\{1, \ldots, J\}$.

\subsubsection{Traders with Different Savings Rates}

If savings rates are different across traders, by theorem 1 , trader $i$ dominates on those sample paths where:

$$
\lim _{t \rightarrow \infty} \inf \sum_{\tau=1}^{t}\left[I_{p}\left(\pi_{\tau}\right)-I_{p}\left(\alpha_{\tau}^{i}\right)+\sum_{s} p_{s} \log \frac{\delta_{s \tau}^{i}}{\delta_{s \tau}}\right]>-\infty
$$

Not surprisingly, other things being equal, the market selects for the most patient investors, i.e. those investors whose savings rate $\delta_{s t}^{i}$ is larger with respect to the average $\delta_{s t}$.

Let us consider now our original problem and namely: what can be said about the asymptotic behaviour of wealth shares in a population of traders who believe in CAPM and of logarithmic utility maximisers? If the savings rate of logarithmic utility maximisers $\left(\delta_{s t}^{L}\right)$ is such that $\delta_{s t}^{L} \geq \delta_{s t}$ a.s., then a first answer to this question is trivial: they will dominate and drive to extinction traders who believe in CAPM.

Proposition 11 Under assumptions 1 and 4, in a population of traders who believe in CAPM and of logarithmic utility maximisers, the latter dominate, provided that their savings rate is at least as large as the average savings rate, and drive to extinction the population of traders who believe in CAPM. Formally, if $\delta_{s t}^{L} \geq \delta_{s t}$ a.s. $\forall s \in\{1, \ldots, S\}, \forall t>0$, then $\lim _{t \rightarrow \infty} \varepsilon_{t}=1$.

\footnotetext{
${ }^{27}$ We need $\gamma_{t}^{j}$ to be either an increasing function or a decreasing function of each trader's wealth level, for all $j$.
} 
Proof. See appendix.

However, by assuming that $\delta_{s t}^{L} \geq \delta_{s t}$ a.s., we actually ignore the fact that logarithmic utility maximisers have a "comparative advantage" from an evolutionary point of view with respect to any other trader, in that they adopt the fittest portfolio rule. In what follows, we prove the dominance of logarithmic utility maximisers under a weaker assumption on savings rates.

We can in fact prove that logarithmic utility maximisers dominate on those sample paths where:

$$
\sum_{s=1}^{S} \frac{\alpha_{s t}^{C A P M} \delta_{s t}^{C A P M}}{\delta_{s t}^{L}} \leq 1
$$

As we suggested, this condition is weaker than requiring $\delta_{s t}^{L} \geq \delta_{s t}$. Namely:

$$
\delta_{s t}^{L} \geq \delta_{s t} \text { a.s. } \Rightarrow \sum_{s=1}^{S} \frac{\alpha_{s t}^{C A P M} \delta_{s t}^{C A P M}}{\delta_{s t}^{L}} \leq 1 \text { a.s. }
$$

while the converse is not true.

Proposition 12 Under assumptions 1 and 4, in a population of traders who believe in $C A P M$ and traders who are endowed with a logarithmic utility function, the latter dominate and drive $C A P M$ traders to extinction if:

$$
\sum_{s=1}^{S} \frac{\alpha_{s t}^{C A P M} \delta_{s t}^{C A P M}}{\delta_{s t}^{L}} \leq 1 \quad \text { a.s. }
$$

Proof. See appendix.

It should be noted that condition 42 is not the weakest one could impose. However, proposition 12 shows that the condition $\delta_{s t}^{L} \geq \delta_{s t}$ a.s. in Blume and Easley (1992) can be relaxed.

\section{Genuine Mean-Variance Behaviour}

Traders who believe in CAPM do not display a genuine mean-variance behaviour: they know what the two-fund separation theorem prescribes, believe it works in reality and only partially optimise in their choice between the risk-free and the market portfolios.

In this section we consider a population of rational traders endowed with mean-variance preferences and we show that, in an evolutionary framework, they will not do any better than traders who believe in CAPM and use what the model prescribes as a rule of thumb.

\subsection{Existence of Equilibrium}

Suppose that there are two types of rational traders in the market: traders who are endowed with a quadratic utility function (and therefore display a genuine mean-variance behaviour) and traders who are endowed with a logarithmic utility function. Given their level of wealth and market prices, traders choose their optimal portfolio composition in the available assets. 
A first question we ask is whether a market clearing equilibrium always exists in this economy. From an analytical point of view, the equilibrium existence problem in this setting is equivalent to the general equilibrium existence problem in a pure exchange economy.

Definition 13 At each date $t \geq 0$, an equilibrium for this economy is an array of portfolio compositions $\left\{q_{t}^{i}\right\}_{i=M V, L O G} \in \Delta^{S \times 2-1}$ and a price vector $\rho_{t} \in \Delta^{S-1}$ such that:

$$
q_{t}^{i}=\arg \max \left\{E\left[u^{i}\left(q_{s t}^{i} w_{s}\right)\right] \quad \text { s.t. } \sum_{s=1}^{S} \rho_{s t} q_{s t}^{i} \leq w_{t-1}^{i}\right\}, \quad \forall i=\{M V, L O G\}
$$

and markets clear:

$$
\sum_{i=M V, L O G} q_{s t}^{i}=1, \quad \forall s \in\{1,2, \ldots, S\}
$$

This is clearly not a pure exchange economy: traders are not endowed with assets' shares but with exogenous wealth. They only express demands, while supply is exogenous. However, we can consider $\left(\varepsilon_{t-1}^{i}, \varepsilon_{t-1}^{i}, \ldots, \varepsilon_{t-1}^{i}\right) \in \Delta^{S-1}$ as if it was an endowment vector in assets' shares for trader $i$ and we can study equilibrium existence as if we were facing a pure exchange general equilibrium model.

We can therefore state the following:

Proposition 14 When there are two types of traders - traders who are endowed with a logarithmic utility function (traders of type $L$ ) and traders who display a genuine meanvariance behaviour (traders of type $M V$ ) - there always exists an equilibrium.

Proof. See appendix.

We can actually characterise equilibrium prices, and prove the following:

Proposition 15 Equilibrium prices have a strictly positive lower bound. Formally, $\exists \nu>$ $0 \mid \pi_{s t} \geq \nu, \forall s \in\{1, \ldots, S\}$ and $\forall t \geq 0$.

Proof. See appendix.

\subsection{The Evolution of Wealth Shares}

Recall that a rational trader endowed with a quadratic utility function chooses a portfolio:

$$
\alpha_{s t}^{M V}=\frac{\pi_{s t}}{w_{s}\left(1-\varepsilon_{t-1}\right) a}-\frac{k_{t}-\left(1-\varepsilon_{t-1}\right) a}{a h_{t} p_{s} w_{s}^{2}\left(1-\varepsilon_{t-1}\right)} \pi_{s t}^{2}
$$

where $\varepsilon_{t-1}$ is the wealth share of logarithmic utility maximisers at (the beginning of) date $t$.

Proposition 15 allows us to claim that the portfolio rules of traders who display a genuine mean-variance behaviour are bounded away from zero. Therefore theorem 1 applies.

Proposition 16 Under assumption 1 and assuming that $\delta_{s t}^{L} \geq \delta_{s t}^{M V}$ a.s. for all $s$ and $t$, in a population of logarithmic utility maximisers and of traders who display a genuine mean variance behaviour, the former dominate and determine asset prices asymptotically. Formally: $\liminf \operatorname{in}_{t \rightarrow \infty} \varepsilon_{t}>0$ and $\pi_{t} \rightarrow p$ almost surely. 
Proof. By proposition 15 and theorem 1.

Our next result shows that mean-variance traders vanish almost surely.

Proposition 17 Under assumption 1 and assuming that $\delta_{s t}^{L} \geq \delta_{s t}^{M V}$ a.s. for all $s$ and $t$, a population of traders who display mean-variance behaviour will be driven to extinction by traders who behave as logarithmic utility maximisers. Formally: $\lim _{\sup _{t \rightarrow \infty}} \varepsilon_{t}^{M V}=0$ almost surely.

Proof. See appendix.

The proof of proposition 17 relies on two steps. We first show (lemma 20 in the appendix) that, in presence of aggregate uncertainty, $\alpha_{s t}^{M V}$ a.s. will not converge to probabilities. Namely:

$$
\operatorname{Pr}\left\{\omega \in \Omega \mid \alpha_{s t}^{M V} \rightarrow p_{s}\right\}=0 \text { for all } s
$$

We then prove that dominance of logarithmic traders and price convergence to probabilities implies that the wealth share of mean-variance traders must converge to zero almost surely.

Corollary 18 In an economy where some traders display a genuine mean-variance behaviour and others believe in CAPM, both types will be driven to extinction, should a logarithmic utility maximiser enter the market. Formally lim $\sup _{t \rightarrow \infty} \varepsilon_{t}^{M V}=0$ and $\lim \sup _{t \rightarrow \infty} \varepsilon_{t}^{C A P M}=$ 0 almost surely.

The proof of corollary 18 is straightforward since the results we proved in the multipopulation framework apply.

\section{Concluding Remarks}

In the evolutionary setting for a financial market developed in Blume and Easley (1992), we consider three types of traders: traders who "believe" in CAPM and use its predictions as a rule of thumb; traders who are endowed with a quadratic utility function and, therefore, display a genuine mean-variance behaviour; finally, traders who are endowed with a logarithmic utility function and therefore adopt the celebrated Kelly criterion for investment. We are interested in the asymptotic behaviour of the wealth shares of these three types of traders. Following Blume and Easley (1992), we define notions of dominance, survival and extinction in the market through the limiting value of each trader's wealth share. In particular, the wealth share of a dominating trader is asymptotically bounded away from zero, while extinction occurs when the wealth share converges almost surely to zero.

Our main results are obtained in a simple setting where traders have constant and identical savings rates. Under this assumption we are able to prove that logarithmic utility maximisers dominate on any sample path. Furthermore, in presence of aggregate uncertainty, traders who believe in CAPM are driven to extinction.

We then check the robustness of these results removing some of the initial simplifying assumptions. Firstly, we allow for more than two types of traders in the market and prove that, generically, our results are robust in a multipopulation framework. Secondly, we allow for heterogeneity among CAPM traders in their degree of risk aversion and we prove that 
our results are robust in this setting. Finally, we allow for different savings rates across traders. We find that the market selects for the most patient investor. Therefore our results are obviously robust so long as logarithmic utility maximisers are relatively more patient than CAPM traders. We prove, however, that this bound can be "relaxed".

Finally, we deal with an economy populated by genuine mean-variance traders. We show that, if a logarithmic utility maximiser enters the market, he dominates, determines market prices asymptotically and drives to extinction the population of mean-variance traders.

\section{Appendix}

Proof of proposition 2. Define $k_{t}=\sum_{s=1}^{S} \pi_{s t} / w_{s}$. Equation 31 becomes:

$$
\rho_{s t}=\frac{p_{s} \varepsilon_{t-1} w_{t-1} w_{s} k_{t}}{\left[1-\left(1-\varepsilon_{t-1}\right)\left(1-\gamma_{t}\right)\right] w_{s} k_{t}-\left(1-\varepsilon_{t-1}\right) \gamma_{t}}
$$

Divide through by $w_{s}$ and sum over $s$ to get:

$$
F_{t}\left(k_{t}\right) \equiv \sum_{s=1}^{S} \frac{p_{s} \varepsilon_{t-1} w_{t-1}}{\left[1-\left(1-\varepsilon_{t-1}\right)\left(1-\gamma_{t}\right)\right] w_{s} k_{t}-\left(1-\varepsilon_{t-1}\right) \gamma_{t}}=1
$$

A solution for $k_{t}$ to:

$$
F_{t}\left(k_{t}\right)=\sum_{s=1}^{S} f_{s t}\left(k_{t}\right)=1
$$

will be market clearing. Assuming that $\varepsilon_{t-1}>0$, there is only one value for $k_{t}$ such that equation 47 holds and prices are non-negative. The graph of $F_{t}\left(k_{t}\right)$ is shown in figure 2 . There are $S$ vertical asymptotes, respectively in

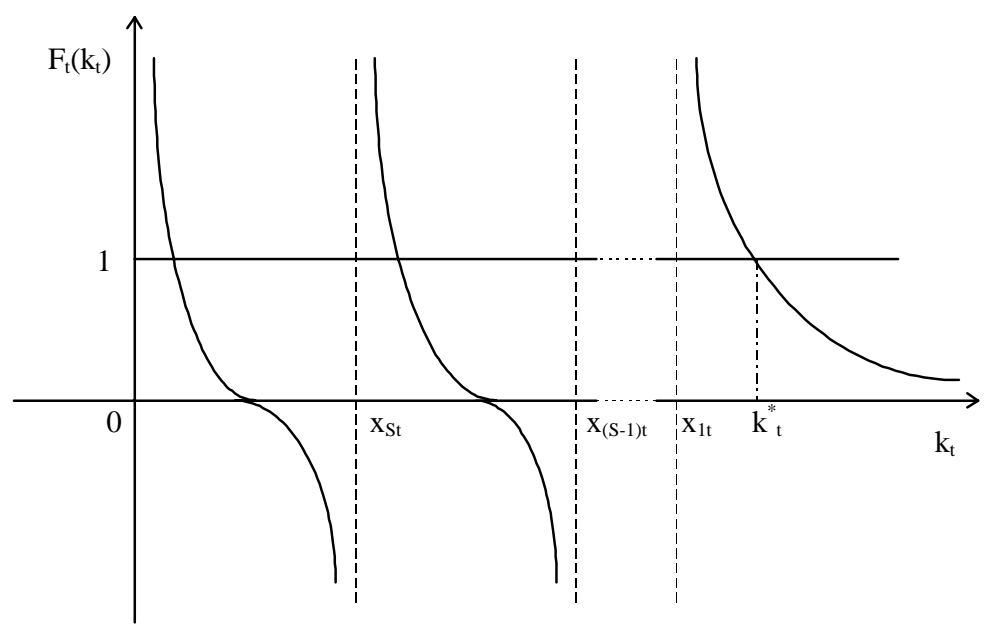

Figure 2: Market clearing. 


$$
x_{s t} \equiv \frac{\left(1-\varepsilon_{t-1}\right) \gamma_{t}}{\left[1-\left(1-\varepsilon_{t-1}\right)\left(1-\gamma_{t}\right)\right] w_{s}}
$$

for each $s \in\{1,2, \ldots, S\}$. Non-negativity of prices requires:

$$
k_{t}>\frac{\left(1-\varepsilon_{t-1}\right) \gamma_{t}}{\left[1-\left(1-\varepsilon_{t-1}\right)\left(1-\gamma_{t}\right)\right] w_{s}} \quad \forall s \in\{1, \ldots, S\}
$$

Therefore:

$$
k_{t}>\frac{\left(1-\varepsilon_{t-1}\right) \gamma_{t}}{\left[1-\left(1-\varepsilon_{t-1}\right)\left(1-\gamma_{t}\right)\right] w_{1}}=x_{1 t}
$$

In the open interval

$$
\left(\frac{\left(1-\varepsilon_{t-1}\right) \gamma_{t}}{\left[1-\left(1-\varepsilon_{t-1}\right)\left(1-\gamma_{t}\right)\right] w_{1}}, \infty\right)
$$

$F_{t}\left(k_{t}\right)$ is monotonic decreasing between $\infty$ and 0 , so that there is a unique value $0<k_{t}^{*}<\infty$ such that 47 holds and prices are non-negative. So long as $\varepsilon_{t-1}>0$, such a value for $k_{t}$ will always exist. On the other hand, if $\varepsilon_{t-1}=0$ then equation 47 cannot be satisfied. Finally:

$$
\varepsilon_{t}=\prod_{\tau=1}^{t} \prod_{s=1}^{S} \iota_{s \tau} \frac{p_{s}}{\pi_{s \tau}} \varepsilon_{0} \geq \varepsilon_{0}, \forall t \geq 0
$$

so that, if $\varepsilon_{0}>0$, then $\varepsilon_{t}>0, \forall t$.

Proof of proposition 3. To characterise equilibrium prices as $\varepsilon_{t} \rightarrow 0$, we need to know the value of $\lim _{\varepsilon_{t} \rightarrow \mathbf{0}} k_{t}$. Notice that, by definition of $k_{t}$ and market clearing, we know that:

$$
\frac{1}{w_{S}} \leq \lim _{\varepsilon_{t} \rightarrow \mathbf{0}} k_{t} \leq \frac{1}{w_{1}}
$$

On the other hand, non-negativity of prices requires:

$$
\lim _{\varepsilon_{t} \rightarrow \mathbf{0}} \frac{p_{s} \varepsilon_{t-1} w_{t-1} w_{s} k_{t}}{\left[1-\left(1-\varepsilon_{t-1}\right)\left(1-\gamma_{t}\right)\right] w_{s} k_{t}-\left(1-\varepsilon_{t-1}\right) \gamma_{t}} \geq 0, \forall s \in\{1, \ldots, S\}
$$

Therefore:

$$
\lim _{\varepsilon_{t} \rightarrow \mathbf{0}} k_{t} \geq \lim _{\varepsilon_{t} \rightarrow \mathbf{0}} \frac{\left(1-\varepsilon_{t-1}\right) \gamma_{t}}{\left[1-\left(1-\varepsilon_{t-1}\right)\left(1-\gamma_{t}\right)\right] w_{1}}=\frac{1}{w_{1}}
$$

The only value for $\lim _{\varepsilon_{t} \rightarrow \mathbf{0}} k_{t}$ that satisfies both 53 and 55 is:

$$
\lim _{\varepsilon_{t} \rightarrow \mathbf{0}} k_{t}=\frac{1}{w_{1}}
$$

which, in turn, implies $\lim _{\varepsilon_{t} \rightarrow \mathbf{0}} \pi_{t}=(1,0, \ldots, 0)$.

Proof of corollary 4. Recall that:

$$
\begin{aligned}
q_{s t}^{L} & =\frac{p_{s} \varepsilon_{t-1} w_{t-1}}{\rho_{s t}}= \\
& =\frac{\left[1-\left(1-\varepsilon_{t-1}\right)\left(1-\gamma_{t-1}\right)\right] w_{s} k_{t}-\left(1-\varepsilon_{t-1}\right) \gamma_{t}}{w_{s} k_{t}}
\end{aligned}
$$


Therefore:

$$
\lim _{\varepsilon_{t} \rightarrow \mathbf{0}} q_{s t}^{L}=\frac{\gamma_{t} w_{s} / w_{1}-\gamma_{t}}{w_{s} / w_{1}}=\gamma_{t}-\gamma_{t} \frac{w_{1}}{w_{s}}
$$

Recall now that:

$$
q_{s t}^{C A P M}=\left(1-\varepsilon_{t-1}\right) \gamma_{t} \frac{1}{w_{s} k_{t}}+\left(1-\varepsilon_{t-1}\right)\left(1-\gamma_{t}\right)
$$

so that, finally:

$$
\lim _{\varepsilon_{t} \rightarrow \mathbf{0}} q_{s t}^{C A P M}=1-\gamma_{t}+\gamma_{t} \frac{w_{1}}{w_{s}}
$$

Lemma 19 Under assumptions 1 and 3, the wealth share of logarithmic utility maximisers $\varepsilon_{t}$ is a submartingale, so that $E\left[\varepsilon_{t} \mid \Im_{t-1}\right] \geq \varepsilon_{t-1}$ for all $t \geq 0$.

Proof of lemma 19. If state $s$ occurs at date $t$, the wealth share of $L$ traders can be expressed as follows:

$$
\varepsilon_{t}=\frac{p_{s}}{\pi_{s t}} \varepsilon_{t-1}
$$

Consider, now, $E\left[\log \varepsilon_{t} \mid \Im_{t-1}\right]=\sum_{s=1}^{S} p_{s} \log p_{s} / \pi_{s t}+\log \varepsilon_{t-1} \geq \log \varepsilon_{t-1}$. Since $\sum_{s=1}^{S} p_{s} \log p_{s} / \pi_{s t}$ is always non-negative and $\log \varepsilon_{t}$ is a concave and increasing function of $\varepsilon_{t}$, by Jensen's inequality and simple algebra it follows that:

$$
E\left[\varepsilon_{t} \mid \Im_{t-1}\right] \geq \varepsilon_{t-1}, \forall t \geq 0
$$

i.e. the process $\left\{\varepsilon_{t}, t \geq 0\right\}$ is a submartingale.

Proof of proposition 5. By lemma 19 and the convergence theorem for submartingales we know that:

$$
\operatorname{Pr}\left\{\exists \varepsilon^{*}|\forall \xi>0, \exists T>0, \forall t \geq T,| \varepsilon_{t}-\varepsilon^{*} \mid<\xi\right\}=1
$$

or, equivalently, $\varepsilon_{t} \rightarrow \varepsilon^{*}$ almost surely. Call $A \subseteq \Omega$ the set of sample paths $\omega \in \Omega$ such that, on $\omega, \varepsilon_{t}$ converges; call $B \subseteq \Omega$ the set of sample paths $\omega \in \Omega$ such that, on $\omega$, each state $s \in\{1, \ldots, S\}$ occurs infinitely often; call $C \equiv A \cap B$. Clearly: $\operatorname{Pr}(A)=1, \operatorname{Pr}(B)=1$ and $\operatorname{Pr}(C)=1$. To prove our claim it is sufficient to show that $\forall \omega \in C, \lim _{t \rightarrow \infty} \varepsilon_{t}(\omega)>0$. By way of contradiction suppose that $\exists \widehat{\omega} \in C$ such that:

$$
\lim _{t \rightarrow \infty} \varepsilon_{t}(\widehat{\omega})=0
$$

This implies that, given $\widehat{\omega}$ :

$$
\lim _{t \rightarrow \infty} \frac{p_{s}}{\pi_{s t}} \varepsilon_{t-1}=0
$$

Recall that $q_{s t}^{L}=\left(p_{s} / \pi_{s t}\right) \varepsilon_{t-1}$ and that by corollary 4 :

$$
\lim _{\varepsilon_{t} \rightarrow \mathbf{0}} q_{s t}^{L}=\gamma_{t}-\gamma_{t} \frac{w_{1}}{w_{s}}
$$


Given $\widehat{\omega}$, consider now a subsequence $t_{\mu}$ such that $\omega_{t_{\mu}} \neq 1 \forall \mu$. (Since $\widehat{\omega} \in C$ the subsequence $t_{\mu}$ is infinite). Using 66 , since we are assuming that 64 holds, we obtain that for any $s \neq 1$ :

$$
\lim _{\mu \rightarrow \infty} \frac{p_{s}}{\pi_{s t_{\mu}}} \varepsilon_{t_{\mu}-1}>0
$$

which contradicts 65 .

Proof of proposition 6. Recall that, if state $s$ occurred at date $t$, then:

$$
\varepsilon_{t}(\omega)=\frac{p_{s}}{\pi_{s t}(\omega)} \varepsilon_{t-1}(\omega)
$$

or, equivalently:

$$
\frac{\varepsilon_{t}(\omega)}{\varepsilon_{t-1}(\omega)}=\frac{p_{s}}{\pi_{s t}(\omega)}
$$

Since $\varepsilon_{t}$ converges almost surely and since $\left\{\omega \in \Omega \mid \varepsilon_{t}(\omega) \rightarrow 0\right\}$ has measure zero, then:

$$
\frac{\varepsilon_{t}(\omega)}{\varepsilon_{t-1}(\omega)} \rightarrow 1 \text { a.s. }
$$

which, finally, implies: $\pi_{s t}(\omega) \rightarrow p_{s}$ a.s.

Proof of proposition 7. Market clearing requires:

$$
\pi_{s t}=\varepsilon_{t} p_{s}+\left(1-\varepsilon_{t-1}\right)\left[\gamma_{t} \alpha_{s t}^{F}+\left(1-\gamma_{t}\right) \pi_{s t}\right]
$$

Define:

$$
\beta_{t} \equiv \frac{\left(1-\varepsilon_{t-1}\right) \gamma_{t}}{\left(1-\varepsilon_{t-1}\right) \gamma_{t}+\varepsilon_{t-1}}
$$

and therefore rewrite equation 71 as:

$$
\pi_{s t}=p_{s}+\beta_{t}\left(\alpha_{s t}^{F}-p_{s}\right)
$$

Recall that $L$ traders dominate and, by proposition $6: \pi_{s t} \rightarrow p_{s}$ almost surely. By eq. 73 we obtain that:

$$
\pi_{s t}-p_{s}=\beta_{t}\left(\alpha_{s t}^{F}-p_{s}\right)
$$

and since $\pi_{s t}-p_{s} \rightarrow 0$ a.s. we need $\beta_{t}\left(\alpha_{s t}^{F}-p_{s}\right) \rightarrow 0$ almost surely as well. Note that $\left(\alpha_{s t}^{F}-p_{s}\right)$ converges almost surely to

$$
\frac{p_{s} / w_{s}}{\sum_{z=1}^{S} p_{z} / w_{z}}-p_{s}
$$

which is different from zero unless there is no aggregate uncertainty. Therefore, we will have $\beta_{t}\left(\alpha_{s t}^{F}-p_{s}\right) \rightarrow 0$ a.s. iff $\beta_{t} \rightarrow 0$ almost surely. By equation 72 :

$$
\left(1-\varepsilon_{t-1}\right) \gamma_{t} \beta_{t}+\varepsilon_{t-1} \beta_{t}=\left(1-\varepsilon_{t-1}\right) \gamma_{t}
$$


If $\beta_{t} \rightarrow 0$ a.s., then, since $\left(1-\varepsilon_{t-1}\right)$ and $\gamma_{t}$ are bounded, $\left(1-\varepsilon_{t-1}\right) \gamma_{t} \beta_{t}+\varepsilon_{t-1} \beta_{t} \rightarrow 0$ a.s. as well and therefore:

$$
\left(1-\varepsilon_{t-1}\right) \gamma_{t} \rightarrow 0 \text { a.s. }
$$

Call $A \subseteq \Omega$ the set of sample paths $\omega \in \Omega$ such that, on $\omega, \varepsilon_{t}$ converges; call $B \subseteq \Omega$ the set of sample paths $\omega \in \Omega$ such that, on $\omega$, each state $s \in\{1, \ldots, S\}$ occurs infinitely often; call $D \subseteq \Omega$ the set of sample paths $\omega \in \Omega$ such that, on $\omega,\left(1-\varepsilon_{t-1}\right) \gamma_{t} \rightarrow 0$; call $E \equiv A \cap B \cap D$. Clearly: $\operatorname{Pr}(A)=\operatorname{Pr}(B)=\operatorname{Pr}(D)=\operatorname{Pr}(E)=1$. To prove our claim it is sufficient to show that $\forall \omega \in E, \lim _{t \rightarrow \infty} \varepsilon_{t}(\omega)=1$. By way of contradiction suppose that $\exists \widehat{\omega} \in E$ such that $\varepsilon^{*}(\widehat{\omega}) \neq 1$. In order to have $\left(1-\varepsilon_{t-1}\right) \gamma_{t} \rightarrow 0$, we need $\gamma_{t}(\widehat{\omega}) \rightarrow 0$. By assumption $4, \gamma_{t}\left(w_{t-1}^{C A P M}\right)$ is a monotonic function of $w_{t-1}^{C A P M}$. Call $\bar{w}$ that level of wealth such that $\gamma_{t}(\bar{w})=0$. If $\gamma_{t}(\widehat{\omega}) \rightarrow 0$, then equivalently $w_{t-1}^{C A P M}(\widehat{\omega}) \rightarrow \bar{w}$. Recall that $w_{t-1}^{C A P M}(\widehat{\omega})=$ $\left[1-\varepsilon_{t-1}(\widehat{\omega})\right] w_{t-1}(\widehat{\omega})$. Therefore, if $\gamma_{t}(\widehat{\omega}) \rightarrow 0$, then $\left[1-\varepsilon_{t-1}(\widehat{\omega})\right] w_{t-1}(\widehat{\omega}) \rightarrow \bar{w}$. We know that $\varepsilon_{t}(\widehat{\omega})$ converges to $\varepsilon^{*}(\widehat{\omega})>0$. However $w_{t}$, in presence of aggregate risk, does not converge. As a result $\gamma_{t}(\widehat{\omega})$ does not converge to 0 . Finally, this implies that, in order to have $\left[1-\varepsilon_{t-1}(\widehat{\omega})\right] \gamma_{t}(\widehat{\omega}) \rightarrow 0$ we need $\left[1-\varepsilon_{t-1}(\widehat{\omega})\right] \rightarrow 0$ which contradicts $\varepsilon^{*}(\widehat{\omega}) \neq 1$.

Proof of proposition 8. Write the portfolio rule of CAPM traders as follows:

$$
\alpha_{s t}^{C A P M}=\gamma_{t} \frac{\pi_{s t} / w_{s}}{k_{t}}+\left(1-\gamma_{t}\right) \pi_{s t}=m_{s t} \pi_{s t}
$$

where $k_{t}=\sum_{s=1}^{S} \pi_{s t} / w_{s}$ and $m_{s t} \equiv \gamma_{t} / w_{s} k_{t}+\left(1-\gamma_{t}\right)$. Market clearing requires:

$$
\pi_{s t}=\varepsilon_{t-1}^{C A P M} \alpha_{s t}^{C A P M}+\varepsilon_{s t}^{L} p_{s}+\sum_{n=1}^{N} \varepsilon_{t-1}^{n} \alpha_{s t}^{n}
$$

which can be rewritten as follows:

$$
\pi_{s t}=\frac{\varepsilon_{t-1}^{L} p_{s}+\sum_{n=1}^{N} \varepsilon_{t-1}^{n} \alpha_{s t}^{n}}{1-\varepsilon_{t-1}^{C A P M} m_{s t}}
$$

Recall that:

$$
\varepsilon_{t}^{L}=\frac{p_{s}}{\pi_{s t}} \varepsilon_{t-1}^{L}=p_{s} \frac{1-\varepsilon_{t-1}^{C A P M} m_{s t}}{\varepsilon_{t-1}^{L} p_{s}+\sum_{n} \varepsilon_{t-1}^{n} \alpha_{s t}^{n}} \varepsilon_{t-1}^{L}
$$

Define, for $j \in\{L,\{1, \ldots, N\}\}$ :

$$
l_{t-1}^{j} \equiv \frac{\varepsilon_{t-1}^{j}}{1-\varepsilon_{t-1}^{C A P M}}
$$

so that equation 81 becomes:

$$
\varepsilon_{t}^{L}=\frac{p_{s} l_{t-1}^{L}\left(1-\varepsilon_{t-1}^{C A P M} m_{s t}\right)}{l_{t-1}^{L} p_{s}+\sum_{n} l_{t-1}^{n} \alpha_{s t}^{n}}=\frac{p_{s}\left(1-\varepsilon_{t-1}^{C A P M} m_{s t}\right)}{p_{s}+\sum_{n}\left(l_{t-1}^{n} / l_{t-1}^{L}\right) \alpha_{s t}^{n}}
$$

Call $A \subseteq \Omega$ the set of sample paths $\omega$ such that, on $\omega \in A, \varepsilon_{t}$ converges; call $B \subseteq \Omega$ the set of sample paths $\omega$ such that, on $\omega \in B$, all states occur infinitely often. Clearly $\operatorname{Pr}(A)=\operatorname{Pr}(B)=\operatorname{Pr}(C \equiv A \cap B)=1$. To prove our claim it is sufficient to show that, 
$\forall \omega \in C, \lim _{t \rightarrow \infty} \varepsilon_{t}(\omega)>0$. By way of contradiction suppose that for some $\widehat{\omega} \in C, \varepsilon_{t}(\widehat{\omega}) \rightarrow 0$. This requires:

$$
\begin{aligned}
& \text { either }\left(1-\varepsilon_{t-1}^{C A P M}(\widehat{\omega}) m_{s t}(\widehat{\omega})\right) \rightarrow 0 \\
& \text { or } \quad \frac{l_{t-1}^{L}(\widehat{\omega})}{l_{t-1}^{n}(\widehat{\omega})}=\frac{\varepsilon_{t-1}^{L}(\widehat{\omega})}{\varepsilon_{t-1}^{n}(\widehat{\omega})} \rightarrow 0 \text { for some } n
\end{aligned}
$$

Case 85 is ruled out by corollary 4.1 in Blume and Easley (1992), p. 20, which claims that ${ }^{28}$ $\liminf _{t \rightarrow \infty} \varepsilon_{t-1}^{L} / \varepsilon_{t-1}^{n}>0$. Case 84 can be ruled out by what writing that:

$$
\left(1-\varepsilon_{t-1}^{C A P M}\right)+\varepsilon_{t-1}^{C A P M} \gamma_{t}\left(1-\frac{1}{w_{s} k_{t}}\right) \rightarrow 0
$$

Now, since when $\varepsilon_{t}^{L} \rightarrow 0$, by corollary 4.1 in Blume and Easley (1992), also $\varepsilon_{t}^{n} \rightarrow 0, \forall n$, then clearly $\lim _{\varepsilon_{t}^{L} \rightarrow \mathbf{0}}\left(1-\varepsilon_{t-1}^{C A P M}\right)=0$, so that 86 requires either $\gamma_{t} \rightarrow 0$, or $\lim _{\varepsilon_{t}^{L} \rightarrow \mathbf{0}}\left(1-1 / w_{s} k_{t}\right)=$ 0 . The case $\gamma_{t} \rightarrow 0$ has been ruled out in the proof of proposition 7 . The case $\lim _{\varepsilon_{t}^{L} \rightarrow \mathbf{0}}\left(1-1 / w_{s} k_{t}\right)$ $=0$ clearly contradicts aggregate uncertainty. Therefore $\lim _{t \rightarrow \infty} \varepsilon_{t}^{L}(\widehat{\omega})>0$ which contradicts our assumption. Moreover $\varepsilon_{t}^{L} / \varepsilon_{t-1}^{L}=p_{s} / \pi_{s t} \rightarrow 1$.

Proof of proposition 9. Recall that:

$$
\pi_{s t}=\varepsilon_{t-1}^{L} p_{s}+\varepsilon_{t-1}^{C A P M} \alpha_{s t}^{C A P M}+\sum_{n=1}^{N} \varepsilon_{t-1}^{n} \alpha_{s t}^{n}
$$

Define:

$$
\begin{aligned}
a_{s t} & \equiv \alpha_{s t}^{C A P M}-p_{s} \\
b_{s t}^{n} & \equiv \alpha_{s t}^{n}-p_{s}
\end{aligned}
$$

so that equation 87 becomes:

$$
\pi_{s t}-p_{s}=\varepsilon_{t-1}^{C A P M} a_{s t}+\sum_{n=1}^{N} \varepsilon_{t-1}^{n} b_{s t}^{n}
$$

Proposition 8 implies that $\pi_{s t}-p_{s} \rightarrow 0$ a.s. and therefore:

$$
\varepsilon_{t-1}^{C A P M} a_{s t}+\sum_{n=1}^{N} \varepsilon_{t-1}^{n} b_{s t}^{n} \rightarrow 0 \text { a.s. }
$$

We can show that $\varepsilon_{t-1}^{C A P M}$ and $\varepsilon_{t-1}^{n}, \forall n$ converge almost surely. In fact, for $\varepsilon^{j *}$ and $j=$ $C A P M,\{1, \ldots, N\}$, we can prove the following:

$$
\begin{aligned}
E\left[\log \varepsilon_{t}^{j}\right] & =\sum_{s=1}^{S} p_{s} \log \frac{\alpha_{s t}^{j}}{\pi_{s t}}+\log \varepsilon_{t-1}^{j}= \\
& =\log \varepsilon_{t-1}^{j}+\left[\sum_{s=1}^{S} p_{s} \log \frac{p_{s}}{\pi_{s t}}-\sum_{s} p_{s} \log \frac{p_{s}}{\alpha_{s t}^{j}}\right]
\end{aligned}
$$

\footnotetext{
${ }^{28}$ Corollary 4.1 in Blume and Easley (1992), p.20 actually claims that $\lim \inf _{t \rightarrow \infty} \varepsilon_{t-1}^{L} / \varepsilon_{t-1}^{n}>0$ a.s., but then call $F \subseteq \Omega$ the set of sample paths such that $\lim _{i n f} \operatorname{in}_{t \rightarrow \infty} \varepsilon_{t-1}^{L} / \varepsilon_{t-1}^{n}>0$. Clearly $\operatorname{Pr}(F)=1$. Then take $\widehat{\omega} \in(C \cap F)$ and the proof follows.
} 
Note that $\sum_{s=1}^{S} p_{s} \log p_{s} / \pi_{s t} \rightarrow 0$ a.s. and that $\sum_{s=1}^{S} p_{s} \log p_{s} / \alpha_{s t}^{j}$ will always be positive. Therefore, no matter what happens in the short run, in the long run, because of the convergence of prices to probabilities, $\varepsilon_{t}^{j}$ must become a supermartingale. In fact:

$$
\lim _{t \rightarrow \infty}\left[E\left(\log \varepsilon_{t}^{j}\right)-\log \varepsilon_{t-1}^{j}\right]<0 \text { a.s. }
$$

As a result, $\varepsilon_{t}^{j}$ converges $^{29}$. We have therefore established that both $\varepsilon_{t}^{C A P M}$ and $\varepsilon_{t}^{n}, \forall n \in$ $\{1, \ldots, N\}$ converge almost surely. Call $\varepsilon^{C A P M *}$ and $\varepsilon^{n *}, \forall n \in\{1, \ldots, N\}$ respectively their limiting values. With aggregate uncertainty, clearly $\operatorname{Pr}\left\{\omega \in \Omega \mid a_{s t}(\omega) \rightarrow 0\right\}=0$; finally, by assumption 6: $\operatorname{Pr}\left\{\omega \in \Omega \mid b_{s t}^{n}(\omega) \rightarrow 0\right\}=0, \forall n \in\{1, \ldots, N\}$. Therefore 91 requires either $\varepsilon^{C A P M *}=0$ a.s. and $\varepsilon^{n *}=0$ a.s., $\forall n \in\{1, \ldots, N\}$, or:

$$
a_{s t} \rightarrow \sum_{n=1}^{N} \frac{\varepsilon^{n *}}{\varepsilon^{C A P M *}}\left(-b_{s t}^{n}\right) \text { a.s. }
$$

Equivalently: $\varepsilon^{C A P M *} \alpha_{s t}^{C A P M}+\sum_{n=1}^{N} \varepsilon^{n *} \alpha_{s t}^{n} \rightarrow\left(1-\varepsilon^{L *}\right) p_{s}$ a.s.

Proof of proposition 10. We first show that logarithmic utility maximisers dominate. Recall that, if state $s$ occurred at date $t$ :

$$
\varepsilon_{t}(\omega)=\frac{p_{s}}{\pi_{s t}(\omega)} \varepsilon_{t-1}(\omega)
$$

Market clearing requires for all $s \in\{1, \ldots, S\}$ :

$$
\pi_{s t}=\frac{p_{s} \varepsilon_{t-1} w_{s} k_{t}}{w_{s} k_{t}-\sum_{j=1}^{I-1} \varepsilon_{t-1}^{j} \gamma_{t}^{j}-\sum_{j=1}^{I-1} \varepsilon_{t-1}^{j}\left(1-\gamma_{t}^{j}\right) w_{s} k_{t}}
$$

where $k_{t}=\sum_{s} \pi_{s t} / w_{s}$. Therefore equation 95 becomes:

$$
\varepsilon_{t}=\frac{\left[1-\sum_{j=1}^{I-1} \varepsilon_{t-1}^{j}\left(1-\gamma_{t}^{j}\right)\right] w_{s} k_{t}-\sum_{j=1}^{I-1} \varepsilon_{t-1}^{j} \gamma_{t}^{j}}{w_{s} k_{t}}
$$

By lemma 19 we know that $\varepsilon_{t} \rightarrow \varepsilon^{*}$ a.s. and we want to show that $\varepsilon^{*}>0$ almost surely. Call $A \subseteq \Omega$ the set of sample paths $\omega$ such that, on $\omega \in A, \varepsilon_{t}$ converges; call $B \subseteq \Omega$ the set of sample paths $\omega$ such that, on $\omega \in B$, all states $s \in\{1, \ldots, S\}$ occur infinitely often. Clearly $\operatorname{Pr}(A)=\operatorname{Pr}(B)=\operatorname{Pr}(C \equiv A \cap B)=1$. To prove our claim it is sufficient to show that, $\forall \omega \in C, \varepsilon^{*}(\omega)>0$. By way of contradiction, suppose, that for some $\widehat{\omega} \in C, \varepsilon^{*}(\widehat{\omega})=0$. Given $\widehat{\omega}$, then:

$$
1-\sum_{j=1}^{I-1} \varepsilon_{t-1}^{j}\left(1-\gamma_{t}^{j}\right)-\frac{\sum_{j=1}^{I-1} \varepsilon_{t-1}^{j} \gamma_{t}^{j}}{w_{s} k_{t}} \rightarrow 0
$$

\footnotetext{
${ }^{29}$ In fact $\lim _{t \rightarrow \infty}\left[E\left(\log \varepsilon_{t}^{j}\right)-\log \varepsilon_{t-1}^{j}\right]<0$ a.s. implies that with probability equal to $1, \exists T \mid \forall t \geq$ $T: E\left[\log \varepsilon_{t}^{j}\right]<\log \varepsilon_{t-1}^{j}$. Let us consider, then, the process starting at $T$ : by the martingale convergence theorem, it will converge almost surely. Obviously the process starting in $t=0$ will converge a.s. as well and to the same limit.
} 
or, equivalently:

$$
\sum_{j=1}^{I-1} \varepsilon_{t-1}^{j} \gamma_{t}^{j}-\frac{\sum_{j=1}^{I-1} \varepsilon_{t-1}^{j} \gamma_{t}^{j}}{w_{s} k_{t}} \rightarrow 0
$$

Since $\gamma_{t}^{j} \nrightarrow 0$ (see proof of proposition 7 ), this can only be the case if $w_{s} k_{t} \rightarrow 1$. In presence of aggregate uncertainty, this is clearly a contradiction. Dominance of logarithmic utility maximisers implies that market prices converge a.s. to probabilities. In fact, by 95 and by $\lim _{t \rightarrow \infty} \varepsilon_{t}>0$ a.s., we get:

$$
\frac{\varepsilon_{t}(\omega)}{\varepsilon_{t-1}(\omega)}=\frac{p_{s}}{\pi_{s t}(\omega)} \rightarrow 1 \text { a.s. }
$$

We can finally prove that CAPM traders vanish. We can write the price equation as follows:

$$
\begin{aligned}
\pi_{s t} & =\varepsilon_{t-1} p_{s}+\sum_{j} \varepsilon_{t-1}^{j} \alpha_{s t}^{C A P M(j)} \\
& =\varphi_{t-1} p_{s}+\left(1-\varphi_{t-1}\right) \alpha_{s t}^{F}
\end{aligned}
$$

where $\varphi_{t-1}=\varepsilon_{t-1} /\left[\varepsilon_{t-1}+\sum_{j} \varepsilon_{t-1}^{j}\left(1-\gamma_{t}^{j}\right)\right]$. Convergence of prices to probabilities requires:

$$
\left(1-\varphi_{t-1}\right)\left(\alpha_{s t}^{F}-p_{s}\right) \rightarrow 0 \text { a.s. }
$$

We know that $\left(\alpha_{s t}^{F}-p_{s}\right)$ converges but not to zero, therefore eq. 102 requires:

$$
\varphi_{t} \rightarrow 1 \text { a.s. }
$$

so that we need:

$$
\sum_{j} \varepsilon_{t}^{j}\left(1-\gamma_{t}^{j}\right) \rightarrow 0 \text { a.s. }
$$

Since we cannot have ${ }^{30} \gamma_{t}^{j} \rightarrow 0$ for any $j \in\{1, \ldots, J\}$, the asymptotic price equation only holds if $\varepsilon_{t}^{j} \rightarrow 0$ a.s. for all $j \in\{1, \ldots, J\}$.

Proof of proposition 11. If $\delta_{s t}^{L} \geq \delta_{s t}$ a.s., then clearly:

$$
\varepsilon_{t}=\frac{\delta_{s t}^{L} p_{s}}{\delta_{s t} \pi_{s t}} \varepsilon_{t-1} \geq \frac{p_{s}}{\pi_{s t}} \varepsilon_{t-1} \text { a.s. }
$$

Finally, since by proposition $5 \lim _{t \rightarrow \infty}\left(p_{s} / \pi_{s t}\right) \varepsilon_{t-1}>0$ a.s., a fortiori

$$
\lim _{t \rightarrow \infty} \frac{\delta_{s t}^{L} p_{s}}{\delta_{s t} \pi_{s t}} \varepsilon_{t-1}>0 \text { a.s. }
$$

Therefore logarithmic utility maximisers dominate. Price convergence to probabilities can be proved by lemma 19 in Blume and Easley (1992), p.34. Finally, dominance of logarithmic utility maximisers and price convergence to probabilities imply that CAPM traders vanish in the same fashion as in the proof of proposition 7 .

\footnotetext{
${ }^{30}$ See proof of proposition 7.
} 
Proof of proposition 12. We first prove price convergence to probabilities and then dominance of logarithmic utility maximisers and extinction of CAPM traders. By lemma A.1 in Blume and Easley (1992), p.34, prices converge almost surely to probabilities if:

$$
\sum_{s=1}^{S} p_{s} \log \frac{\delta_{s t}^{L} p_{s}}{\delta_{s t} \pi_{s t}} \geq 0, \forall t \geq 0
$$

Recall that:

$$
\delta_{s t}=\frac{\delta_{s t}^{L} p_{s} \varepsilon_{t-1}+\delta_{s t}^{C A P M} \alpha_{s t}^{C A P M}\left(1-\varepsilon_{t-1}\right)}{\pi_{s t}}
$$

so that condition 107 becomes:

$$
\sum_{s=1}^{S} p_{s} \log \frac{p_{s}}{p_{s} \varepsilon_{t-1}+\left(\delta_{s t}^{C A P M} \alpha_{s t}^{C A P M} / \delta_{s t}^{L}\right)\left(1-\varepsilon_{t-1}\right)} \geq 0, \forall t \geq 0 .
$$

We can show that a sufficient condition for the left-hand side of 109 to achieve a non-negative minimum is that:

$$
0<\sum_{s=1}^{S} p_{s} \varepsilon_{t-1}+\sum_{s=1}^{S} \frac{\delta_{s t}^{C A P M} \alpha_{s t}^{C A P M}}{\delta_{s t}^{L}}\left(1-\varepsilon_{t-1}\right) \leq 1
$$

or equivalently:

$$
-\frac{\varepsilon_{t-1}}{1-\varepsilon_{t-1}}<\sum_{s=1}^{S} \frac{\delta_{s t}^{C A P M} \alpha_{s t}^{C A P M}}{\delta_{s t}^{L}} \leq 1
$$

The first constraint does not bind (since the summation will always be positive). Therefore the only binding inequality is the second one. We can finally prove extinction of CAPM traders (and indirectly dominance of logarithmic utility maximisers) similarly to proposition 7 .

Proof of proposition 14. In a pure exchange economy, each trader $i$ has an endowment in assets' shares $\left\{x_{s t}^{i}\right\}_{s=1}^{S}$ and chooses an optimal portfolio solving the following problem:

$$
q_{t}^{i}=\arg \max \left\{E\left[u^{i}\left(q_{s t}^{i} w_{s}\right)\right] \quad \text { s.t. } \sum_{s=1}^{S} \rho_{s t}\left(q_{s t}^{i}-x_{s t}^{i}\right) \leq 0\right\}
$$

Moreover, market clearing requires for all $s \in\{1, \ldots, S\}$ :

$$
\sum_{i=M V, L O G} q_{s t}^{i}=\sum_{i=M V . L O G} x_{s t}^{i}
$$

However, going back to definition 13, it is straightforward to show that problems P1 and P2 are equivalent from an analytical point of view, when one substitutes $\varepsilon_{t-1}^{i}$ for $x_{s t}^{i}$. In fact, since $w_{t-1}=\sum_{s} \rho_{s t}$, problem $\mathrm{P} 1$ can be rewritten as follows:

$$
q_{t}^{i}=\arg \max \left\{E\left[u^{i}\left(q_{s t}^{i} w_{s}\right)\right] \quad \text { s.t. } \sum_{s=1}^{S} \rho_{s t}\left(q_{s t}^{i}-\varepsilon_{t-1}^{i}\right) \leq 0\right\}
$$


Finally, the market clearing condition requires for all $s \in\{1, \ldots, S\}$ :

$$
\sum_{i=M V, L O G} q_{s t}^{i}=\sum_{i=M V . L O G} \varepsilon_{t-1}^{i}=1
$$

Therefore we can consider $\left(\varepsilon_{t-1}^{i}, \varepsilon_{t-1}^{i}, \ldots, \varepsilon_{t-1}^{i}\right) \in \Delta^{S-1}$ as if it was an endowment vector in assets' shares for trader $i$ and we can study equilibrium existence as if we were facing a pure exchange general equilibrium model. Existence is guaranteed by strictly positive endowments and continuous, strongly monotone and strictly convex preferences.

Proof of proposition 15. Recall that demands are as follows:

$$
\begin{aligned}
q_{s t}^{M V} & =\frac{1}{a w_{s}}-\frac{k_{t}-a\left(1-\varepsilon_{t-1}\right)}{a h_{t} p_{s} w_{s}^{2}} \pi_{s t} \\
q_{s t}^{L} & =\frac{\varepsilon_{t-1} p_{s}}{\pi_{s t}}
\end{aligned}
$$

where $k_{t} \equiv \sum_{s=1}^{S} \pi_{s t} / w_{s}$ and $h_{t} \equiv \sum_{s=1}^{S} \pi_{s t}^{2} / p_{s} w_{s}^{2}$. Market clearing requires, $\forall s \in\{1, \ldots, S\}$ :

$$
\frac{k_{t}-a\left(1-\varepsilon_{t-1}\right)}{a h_{t} p_{s} w_{s}^{2}} \pi_{s t}^{2}-\left(\frac{1}{a w_{s}}-1\right) \pi_{s t}-\varepsilon_{t-1} p_{s}=0
$$

Solving for equilibrium prices we find:

$$
\begin{aligned}
\pi_{s t} & =\frac{\left(1 / a w_{s}-1\right)+\sqrt{\left(1 / a w_{s}-1\right)^{2}+4 \varepsilon_{t-1} p_{s}\left[k_{t}-a\left(1-\varepsilon_{t-1}\right)\right] / a h_{t} p_{s} w_{s}^{2}}}{2\left[k_{t}-a\left(1-\varepsilon_{t-1}\right)\right] / a h_{t} p_{s} w_{s}^{2}} \geq \\
& \geq \frac{1 / a w_{s}-1}{\left[k_{t}-a\left(1-\varepsilon_{t-1}\right)\right] / a h_{t} p_{s} w_{s}^{2}}
\end{aligned}
$$

Finally, since both $k_{t}$ and $h_{t}$ are bounded:

$$
\begin{aligned}
\frac{1}{w_{S}} & \leq k_{t} \leq \frac{1}{w_{1}} \\
\frac{1}{\max _{s}\left\{p_{s}\right\} w_{S}^{2}} & \leq h_{t} \leq \frac{1}{\min _{s}\left\{p_{s}\right\} w_{1}^{2}}
\end{aligned}
$$

we find that $\forall s \in\{1, \ldots, S\}, t \geq 0$ :

$$
\pi_{s t}>\left(1-a w_{S}\right) \frac{\min _{s}\left\{p_{s}\right\}}{\max _{s}\left\{p_{s}\right\}}\left(\frac{w_{1}}{w_{S}}\right)^{3}>0
$$

Provided that satiation is not attained, namely provided that $a<1 / w_{S}$, this lower bound is strictly positive.

Lemma 20 In presence of aggregate uncertainty $\alpha_{s t}^{M V}$ almost surely does not converge to probabilities. Formally: if $w_{s} \neq w_{s^{\prime}}$ for all $s, s^{\prime} \in\{1, \ldots, S\}$, then $\operatorname{Pr}\left\{\omega \in \Omega \mid \alpha_{s t}^{M V} \rightarrow p_{s}\right\}=$ 0 . 
Proof of lemma 20. Recall that mean-variance traders' portfolio rules are as follows:

$$
\alpha_{s t}^{M V}=\frac{\pi_{s t}}{a w_{s}\left(1-\varepsilon_{t-1}\right)}-\frac{k_{t}-a\left(1-\varepsilon_{t-1}\right)}{a h_{t} p_{s} w_{s}^{2}\left(1-\varepsilon_{t-1}\right)} \pi_{s t}^{2}
$$

When prices converge to probabilities we get that:

$$
\alpha_{s t}^{M V} \rightarrow \frac{p_{s}}{w_{s}}\left[m_{t}-\frac{n_{t}}{w_{s}}\right] \text { a.s. }
$$

where $m_{t}$ and $n_{t}$ are defined as follows:

$$
\begin{aligned}
m_{t} & \equiv \frac{1}{a\left(1-\varepsilon_{t-1}\right)} \\
n_{t} & \equiv \frac{k_{t}-a\left(1-\varepsilon_{t-1}\right)}{a h_{t}\left(1-\varepsilon_{t-1}\right)}
\end{aligned}
$$

Consider the set $B \subseteq \Omega$ of sample paths $\omega$ such that, on $\omega \in B$, all states occur infinitely often. This set has clearly measure one. By way of contradiction suppose that for some $\widehat{\omega} \in B, \alpha_{s t}^{M V}(\widehat{\omega}) \rightarrow p_{s}$. This implies that, given $\widehat{\omega},\left(p_{s} / w_{s}\right)\left[m_{t}-n_{t} / w_{s}\right]=p_{s}$ or, equivalently, $w_{s}^{2}-m_{t} w_{s}-n_{t}=0$. This equation yields a solution for $w_{s}$ that does not depend on $s$, which contradicts aggregate uncertainty.

Proof of proposition 17. Market clearing requires:

$$
\pi_{s t}=\varepsilon_{t-1} p_{s}+\left(1-\varepsilon_{t-1}\right) \alpha_{s t}^{M V}
$$

or equivalently:

$$
\pi_{s t}-p_{s}=\left(1-\varepsilon_{t-1}\right)\left(\alpha_{s t}^{M V}-p_{s}\right)
$$

Dominance of traders with a logarithmic utility function implies:

$$
\pi_{s t} \rightarrow p_{s} \text { a.s. }
$$

and therefore:

$$
\left(1-\varepsilon_{t-1}\right)\left(\alpha_{s t}^{M V}-p_{s}\right) \rightarrow 0 \text { a.s. }
$$

By lemma 20, $\left(\alpha_{s t}^{M V}-p_{s}\right) \nrightarrow 0$. As a result, $\left(1-\varepsilon_{t}\right) \rightarrow 0$ a.s. and $\varepsilon_{t} \rightarrow 1$ a.s. 


\section{REFERENCES}

Algoet, Paul H., and Thomas M.Cover, 1988, Asymptotic Optimality and Asymptotic Equipartition Properties of Log-Optimum Investment, Annals of Probability16, 876-898.

Biais, Bruno, and Raphael Shadur, 1994, On the Survival of Irrational Traders: A Darwinian Approach, Working Paper 94/32 IDEI Toulouse.

Becker, Gary S., 1962, Irrational Behavior and Economic Theory, Journal of Political Economy 70, 1-13.

Blume, Lawrence E., and David Easley, 1992, Evolution and Market Behaviour, Journal of Economic Theory 58, 9-40.

Blume, Lawrence E., and David Easley, 1993, Economic Natural Selection, Economic Letters 42, 281-289.

Breiman, Leo, 1961, Optimal Gambling Systems for Favorable Games, in: Proceedings of the Fourth Berkeley Symposium (University of California Press).

Canning, David, 1997, Evolution, Preferences, and Choice Under Uncertainty, mimeo, Queen's University of Belfast.

Cohn, Richard A.,Wilbur G. Lewellen, Ronald C. Lease, and Gary G. Schlarbaum, 1975, Individual Investor Risk Aversion and Investment Portfolio Composition, Journal of Finance $2,605-620$.

Cooper, William S., and Robert H. Kaplan, 1982, Adaptive 'Coin-Flipping': a Decisiontheoretic Examination of Natural Selection for Random Individual Variation, Journal of Theoretical Biology 94, 135-151.

De Long, J.Bradford, Andrei Shleifer, Lawrence H. Summers, and Robert J. Waldmann, 1990, Noise Trader Risk in Financial Markets, Journal of Political Economy 98, 703-738.

De Long, J.Bradford, Andrei Shleifer, Lawrence H. Summers, and Robert J. Waldmann, 1991, The Survival of Noise Traders in Financial Markets, Journal of Business 64, 1-19.

Fama, Eugene F., and Kenneth R. French, 1996a, Multifactor Explanations of Asset Pricing Anomalies, Journal of Finance 51, 55-84.

Fama, Eugene F., and Kenneth R. French, 1996b, The CAPM is Wanted, Dead or Alive, Journal of Finance 51, 1947-1958.

Finkelstein, Mark, and Robert Whitley, 1981, Optimal Strategies for Repeated Games, Advanced Applied Probability 13, 415-428.

Friend, Irvin, and Marshall E. Blume, 1975, The Demand for Risky Assets, American Economic Review 5, 900-922.

Gode, Dhananjay K., and Shyam Sunder, 1993, Allocative Efficiency of Markets with Zero-Intelligence Traders: Market as a Partial Substitute for Individual Rationality, Journal of Political Economy 101, 119-137.

Goldman, M.Barry, 1974, A Negative Report on the 'Near Optimality' of the MaxFxpected-Log Policy as Applied to Bounded Utilities for Long Lived Programs, Journal of Financial Economics 1, 97-103.

Hakansson, Nils H., 1971, Multi-Period Mean-Variance Analysis: Toward a General Theory of Portfolio Choice, Journal of Finance 26, 857-884. 
Jagannathan, Ravi, and Zhenyu Wang, 1996, The Conditional CAPM and the CrossSections of Expected Returns, Journal of Finance 51, 3-53.

Kelly, J.L., 1956, A New Interpretation of Information Rate, Bell System Technical Journal 35, 917-926.

Latane, Henry A., 1959, Criteria of Choice among Risky Ventures, Journal of Political Economy 67, 144-155.

Lintner, John, 1965, The Valuation of Risk Assets and the Selection of Risky Investments in Stock Portfolios and Capital Budgets, Review of Economics and Statistics 43, 13-37.

Luenberger, David G., 1993, A Preference Foundation for Log Mean-Variance Criteria in Portfolio Choice Problems, Journal of Economic Dynamics and Control 17, 887-906.

Luo, GuoYing, 1995, Evolution and Market Competition, Journal of Economic Theory $67,223-250$.

Merton, Robert C., and Paul A. Samuelson, 1974, Fallacy of the Log-Normal Approximation to Optimal Portfolio Decision-Making over Many Periods, Journal of Financial Economics 1, 67-94.

Mossin, Jan, 1966, Equilibrium in a Capital Asset Market, Econometrica 34, 768-783.

Mossin, Jan, 1968, Optimal Multiperiod Portfolio Policies, Journal of Business 41, 215229 .

Penrose, Edith T., 1952, Biological Analogies in the Theory of the Firm, American Economic Review, 804-819.

Plott, Charles R., 1982, Industrial Organization Theory and Experimental Economics, Journal of Economic Literature 20, 1485-1527.

Robson, Arthur J., 1996, A Biological Basis for Expected and Non-expected Utility, Journal of Economic Theory 68, 397-424.

Rogers, Alan R., 1994, Evolution of Time Preference by Natural Selection, American Economic Review 84, 460-481.

Schaffer, Mark E., 1989, Are Profit-Maximisers the Best Survivors?, Journal of Economic Behavior and Organization 12, 29-45.

Sharpe, William F., 1964, Capital Asset Prices: A Theory of Market Equilibrium under Conditions of Risk, Journal of Finance 19, 425-442.

Shefrin, Hersh, and Meir Statman, 1994, Behavioral Capital Asset Pricing Theory, Journal of Financial and Quantitative Analysis 29, 323-349.

Witt, Ulrich, 1986, Firms' Market Behavior under Imperfect Information and Economic Natural Selection, Journal of Economic Behavior and Organization 7, 265-290. 